\title{
Idoneidad del índice SL para la identificación semi-automática de movimientos en masa que afectan a la red de drenaje
}

\author{
G. B. Subiela Blanco(1), M. Guinau i Sellés ${ }^{(1)}$ y J. P. Galve Arnedo(2) \\ (1) Departament de Dinàmica de la Terra i l'Oceà, Facultat de Ciències de la Terra, Universitat de Barcelona, \\ Martí i Franquès, s/n, 08028 Barcelona, España. \\ gbsubiela@hotmail.com, mguinau@ub.edu, \\ (2) Departamento de Geodinámica, Facultad de Ciencias, Universidad de Granada, \\ Avenida de Fuente Nueva, s/n, 18071 Granada, España. \\ jpgalve@gr.es
}

\begin{abstract}
RESUMEN
Los índices geomorfológicos, como el índice del gradiente del perfil longitudinal de los ríos (Stream LengthGradient index, SL) han demostrado ser buenos identificadores de anomalías en los perfiles longitudinales de los ríos. Generalmente, los factores más comunes que producen estas anomalías (knickpoints) son principalmente cambios litológicos, estructuras tectónicas activas y grandes movimientos de ladera. Este estudio se propone detectar zonas de la red de drenaje afectadas por movimientos en masa utilizando el índice SL en las cuencas de La Vall d'Àssua, Romadriu y La Vall de Tor (Pirineos Centrales). La metodología utilizada consiste en calcular el índice SL utilizando la herramienta Chi-Map y, después, se han aplicado las herramientas Análisis de Puntos Calientes (Hotspot and Cluster Analysis, HCA) y Densidad de Kernel (Kernel Density, KD) de ArcGIS ${ }^{\circledR}$ para proporcionar una mejor visualización y localización de los valores anómalos del índice SL. Las anomalías de SL han sido comparadas con el inventario de inestabilidades de ladera existente en la cuenca de La Vall d'Àssua. Los resultados obtenidos de este análisis indican que hay una buena correlación entre anomalías de SL y los movimientos en masa. Además, este índice geomorfológico ha ayudado en la identificación de 13 nuevas inestabilidades de ladera en el conjunto de las tres cuencas analizadas. En conclusión, el estudio realizado ha permitido comprobar la utilidad del índice SL para detectar aquellos puntos de la red de drenaje que están afectados por movimientos en masa.
\end{abstract}

Palabras clave: anomalía, índice SL, knickpoint, movimiento en masa, red de drenaje.

\section{Suitability of the SL index for the semi-automatic identification of mass movements affecting the drainage network}

\begin{abstract}
Geomorphological indices, such as the Stream Length-Gradient (SL) index, have been demonstrated to be suitable for detecting anomalies in stream-profiles. These anomalies (knickpoints) are generally related to active tectonics, lithological changes and large mass movements. This study aims to detect drainage areas affected by mass movements using SL index in La Vall d'Àssua, Romadriu and La Vall de Tor basins (Central Pyrenees). The methodology used consists of computing the SL index using the Chi-Map script and then, applying two ArcGIS ${ }^{\circledR}$ tools, which are the Hotspot and Cluster Analysis (HCA) and Kernel Density (KD), to provide a better visualisation and location of the knickpoints. SL anomalies have been compared with the slope failure inventory of La Vall d'Àssua basin, indicating a good correlation between SL anomalies and slope failures. Additionally, this geomorphological index provided the identification of 13 mass movements which had not previously been described. To sum up, this geomorphological parameter is a useful tool for the identification of drainage network areas affected by mass movements.
\end{abstract}

Keywords: anomaly, drainage network, knickpoint, mass movement, SL index. 


\section{Introducción}

El inventario de movimientos en masa es la fuente de información más importante para el análisis de la susceptibilidad, peligrosidad y el riesgo de estos procesos gravitacionales (Van Westen et al., 2006). Un estudio realizado por Van Den Eeckhaut and Hervás (2012) destacó que, en general, los inventarios de inestabilidades de ladera europeos actuales son mejorables. Por ejemplo, estos autores consideran que la información contenida en la base de datos de movimientos del terreno de Cataluña (LLISCAT) es un $25 \%$ menor a los datos recopilados en los inventarios europeos de inestabilidades de ladera.

En este trabajo se plantea utilizar el índice del gradiente del perfil longitudinal de los ríos (StreamLength Gradient Index, SL) con el fin de conocer su utilidad para completar esos inventarios. Según Hack (1973), este índice morfométrico permite detectar pendientes anómalas (knickpoints) en una sección del perfil longitudinal de un río. El concepto de knickpoint hace referencia a un cambio brusco en la pendiente del perfil longitudinal de un río (Von Engeln, 1940). En algunos estudios publicados en español, el término knickpoint se traduce como punto de inflexión, punto de quiebre o punto de ruptura como en los trabajos de Ortega and Garzón (2008), Castillo and Lugo-Hubp (2011) y Capó and Garcia (2014). Esta anomalía puede estar asociada a cambios litológicos o estructuras tectónicas activas. Se pueden citar los trabajos de Chen et al. (2003), Troiani and Della Seta (2008) y Viveen et al. (2012) en donde el índice SL ha sido utilizado para analizar este tipo de knickpoints y evaluar la actividad tectónica de una región. Recientemente, los estudios realizados por El Hamdouni et al. (2010), Galve et al. (2014) y Troiani et al. $(2014,2017)$ indican que el índice SL puede ser útil para detectar knickpoints asociados a movimientos en masa que han afectado la red de drenaje.

Este trabajo plantea la aplicación del índice SL en tres cuencas de los Pirineos catalanes para determinar la utilidad de este índice en la identificación de inestabilidades de ladera $y$, adicionalmente, si se constata esto, ayudar a completar el inventario de movimientos en masa existente en sectores donde no hay o está incompleta la información sobre inestabilidades de ladera. Para realizar dicho test, se ha aplicado el índice SL en tres cuencas hidrográficas de los Pirineos catalanes con 3 casuísticas diferentes: 1) una donde existe un inventario completo de movimientos en masa; 2 ) otra donde el inventario que existe no es exhaustivo; y 3 ) una tercera donde no existe inventario de inestabilidades de ladera.

\section{Las anomalías y los movimientos de ladera}

El índice SL permite detectar cambios bruscos en la pendiente del perfil longitudinal de un río. Estas anomalías son normalmente mostradas por el índice SL
(A) Caída del nivel de base
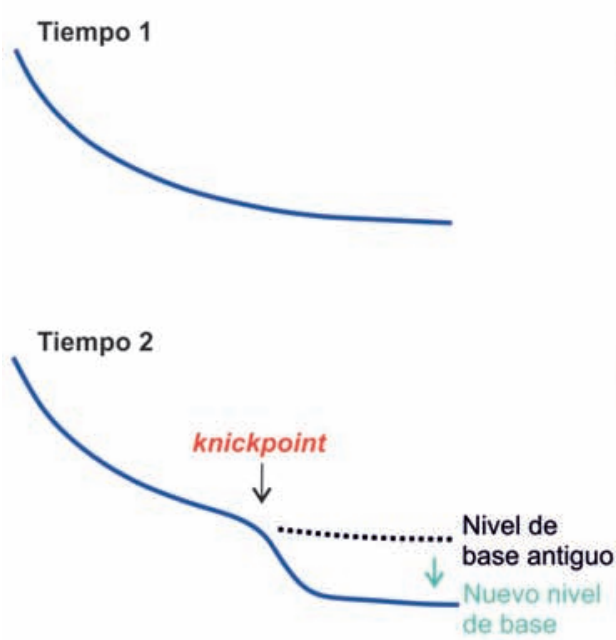

(B) Actividad tectónica
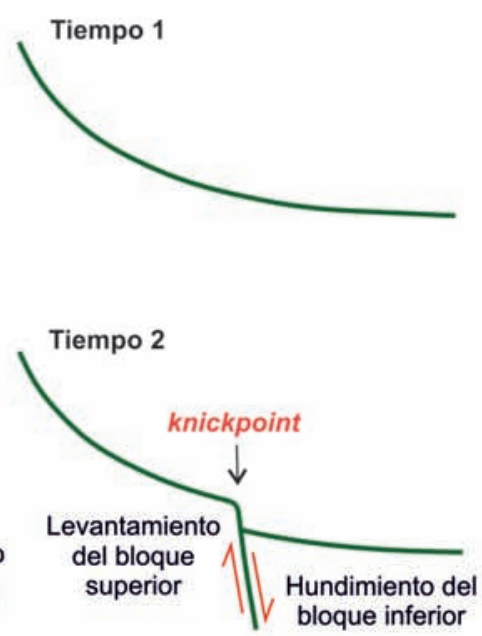

(C) Contraste litológico

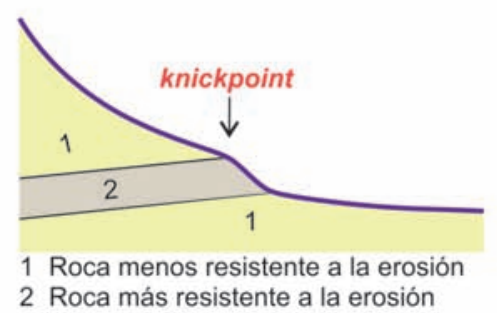

(D) Inestabilidad de ladera

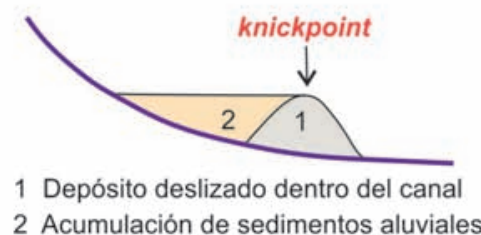

Figura 1. Ejemplos de diferentes factores causantes de anomalías: (A) anomalías asociadas a variaciones en el nivel de base, (B) anomalías asociadas a la actividad tectónica y (C) anomalías asociadas a cambio litológicos, modificados de Bailey (http://wmblogs.wm.edu), y (D) anomalías asociadas a inestabilidades de ladera, modificado de Ouimet et al. (2007).

Figure 1. Examples of factors causing anomalies: (A) anomalies associated with variations in the base level, (B) anomalies associated with tectonic activity and $(C)$ anomalies associated with lithological changes and (D) anomalies associated with slope failures. The first three examples are modified from Bailey (http://wmblogs.wm.edu) and the last one is modified from Ouitmet et al. (2007). 
mediante valores anómalamente altos. Los factores más comunes que producen estos knickpoints son principalmente: variaciones en el nivel de base, cambios litológicos, fallas activas e inestabilidades de ladera (Troiani et al. 2014) (Fig. 1).

En el caso de los movimientos en masa, el knickpoint se genera habitualmente debido a la acumulación de material movilizado en el lecho del canal. En este tramo el río pierde energía, sedimenta la carga sólida que transportaba y forma un rellano aguas arriba. Por lo tanto, el depósito de material deslizado contribuye a la modificación de la morfología del canal $y$, en consecuencia, de su perfil longitudinal (Ouimet et al., 2007).

No obstante, el impacto que ocasiona un movimiento de ladera en el perfil longitudinal del río depende de: a) la magnitud de dicho deslizamiento; b) del material deslizado; y c) de la capacidad de incisión del río. Por ende, no siempre los movimientos de ladera están asociados a un knickpoint (Ouimet et al., 2007). Además, según Keller and Pinter (2002), la interpretación de los knickpoints a partir de los datos obtenidos por los índices geomorfológicos no es en muchos casos directa, ya que, el mismo proceso geológico pueden causar diferentes anomalías $y$, al mismo tiempo, la misma anomalía puede explicarse por diferentes procesos.

Localizar estas anomalías asociadas a inestabilidades de ladera que han afectado a la red de drenaje tiene una implicación crucial en el ámbito de los riesgos geológicos. El índice SL puede ayudar en la identificación de movimientos en masa en zonas donde estos están perturbando la dinámica natural de un río por desviarlo ligeramente, modificar su perfil o bloquear puntualmente el canal principal de un valle. Este último caso puede originar además situaciones de riesgo dado que la obturación del río puede provocar inundaciones aguas arriba o, también, la rotura repentina de esta presa natural generando inundaciones catastróficas aguas abajo (Ermini and Casagli, 2003; Korup, 2004; Pánek et al., 2007).

\section{Objetivos}

El objetivo general de este estudio consistió en conocer la validez del índice del gradiente del perfil longitudinal del río (Stream-length gradient index, SL), como parámetro adicional que ayude en la identificación de movimientos en masa. Para alcanzar la finalidad principal de este trabajo, se ha planteado comprobar la eficacia de los métodos utilizados para analizar e interpretar los valores de SL. También, como indican otros trabajos previos, investigar si existe una correlación espacial entre valores anómalos de SL y movimientos de ladera. Y si es así, comprobar en un lugar sin datos previos que el índice ayuda a identificar inestabilidades de ladera hasta el momento no documentados. Para cumplir estos objetivos se ha propuesto:

- Aplicar métodos de post-procesado para identificar valores anómalos en el índice SL dentro de las zonas de estudio.

- Estudiar la relación de los knickpoints con la existencia de movimientos en masa u otros elementos que puedan generarlos.

- Aplicar la metodología estudiada para seleccionar zonas en las cuales se pueda completar el inventario de inestabilidades de ladera.

\section{Área de estudio}

El trabajo realizado se ha desarrollado en tres cuencas de torrentes tributarios del río Noguera-Pallaresa. La cuenca del río Noguera-Pallaresa, se localiza al NE de la Península Ibérica, concretamente, en los Pirineos Centrales (Fig. 2A). En esta cuenca hidrográfica de $2.807 \mathrm{~km}^{2}$, el cauce principal nace en el Pla de Beret, en la Vall d'Aran, y prosigue su transcurso atravesando las Sierras Exteriores prepirenaicas hasta desembocar en el río Segre (afluente del Ebro) cerca de Camarasa (Fig. 2B). Dentro de esta cuenca se encuentran las cuencas de estudio: La Vall d'Àssua, Romadriu y La Vall de Tor situadas dentro de la comarca del Pallars Sobirà. Estas cuencas han sido seleccionadas ya que (1) la cuenca de La Vall d'Àssua presenta una cartografía exhaustiva en movimientos en masa realizada por Furdada (1988), (2) la cuenca del Romadriu se caracteriza por un inventario de escarpes asociado a inestabilidades de ladera realizado por el grupo de investigación RISKNAT de la Universitat de Barcelona (UB), parcialmente publicado en Ortuño et al. (2017). Y, finalmente, (3) La Vall de Tor como ejemplo de cuenca donde no hay datos previos sobre estos procesos gravitacionales. Geográficamente, $L a$ Vall d'Àssua se ubica en el margen derecho de la Noguera-Pallaresa y presenta un área de drenaje de $92 \mathrm{~km}^{2}$. Se divide en tres torrentes principales: el barranco de Caregue con una longitud de $10 \mathrm{~km}$, el barranco de Berasti $(8,7 \mathrm{~km})$ y el barranco de Altron ( 9 $\mathrm{km}$ ), que confluyen en el torrente de Sant Antoni (4 $\mathrm{km})$, el cual acaba desembocando a la NogueraPallaresa en Rialp (Fig. 2).

A unos $7 \mathrm{~km}$ hacia al norte y en el margen izquierdo del río Noguera-Pallaresa, se encuentra la cuenca del río Romadriu. Dicha cuenca es alargada, asimétrica y tiene mayor área de drenaje $\left(109,91 \mathrm{~km}^{2}\right)$. Su 

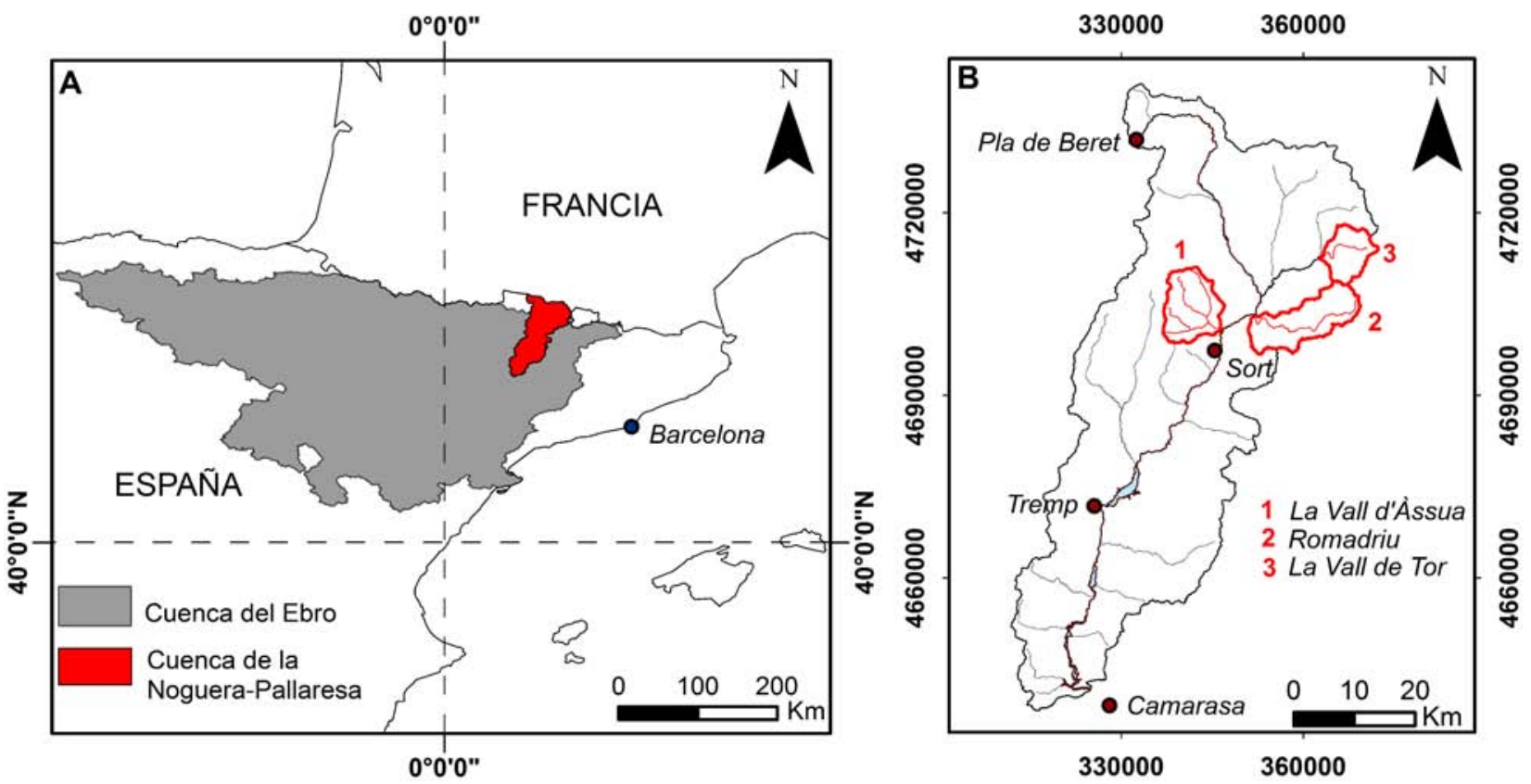

Figura 2. Situación geográfica de las zonas de estudio: La Vall d’Àssua (1), Romadriu (2), La Vall de Tor (3). (C Cuenca Hidrográfica del Ebro, disponible en http://ide-ebro.chebro.es/.

Figure 2. Study areas locations: La Vall d'Àssua (1), Romadriu (2) and La Vall de Tor (3) basins. (c) Ebro basin is available at http://ideebro.chebro.es/.

cauce principal es denominado Romadriu o Santa Magdalena y presenta una dirección predominante EW. Cuenta con 10 torrentes tributarios principales de corta trayectoria (desde 1,4 km en el torrente de la Font de la Freixa en la ladera norte, hasta $5,8 \mathrm{~km}$ en el barranco de la Pega en la ladera sur).

Finalmente, la cuenca de La Vall de Tor se ubica al norte de la cuenca de Romadriu. Esta última zona de estudio presenta un área de drenaje de $55,87 \mathrm{~km}^{2}$, el rio principal es la Noguera deTory sus tributarios son Boixedo y Rabassa, de 3,6 y $4,5 \mathrm{~km}$ de longitud respectivamente (Fig. 2).

\section{Contexto geológico}

El área de estudio se localiza al noreste de la Península Ibérica, en la Zona Axial de los Pirineos. Esta cordillera presenta una doble cresta asimétrica de dirección W-E que se formó durante la orogenia alpina (Cretácico superior hasta inicio del Mioceno), como resultado de la convergencia entre las placas Ibérica y Europea (Muñoz, 1992). La Vall d'Àssua y el Romadriu se encuentran sobre el Domo del Orri y el Sinclinal de Llavorsí, además el sector nor-oriental de la última cuenca se ubica en el Domo de la Pallaresa (Hartevelt, 1970). Asimismo, gran parte de La Vall de Tor se localiza en dicha unidad estructural, mientras que su sector oriental se encuentra en el Sinclinal de Tor (Clariana García, 2015).

Los materiales de la zona de estudio están formados por rocas metasedimentarias de edad CambroOrdovícica, Silúrica y Devónica con un alto grado de esquistosidad (Zandvliet, 1960). Además, en algunas cabeceras de las cuencas de La Vall d'Ássua, Romadriu y La Vall de Tor hay depósitos periglaciales, glaciales, fluviales, de ladera y canchales cuaternarios.

\section{Contexto geomorfológico}

Las principales características geomorfológicas del área de estudio están fuertemente condicionadas por el modelado preglacial y glacial. No obstante, el conjunto de procesos activos fluvio-torrenciales y de ladera han establecido la morfología del relieve actual de las zonas de La Vall de Tor, La Vall d'Àssua y Romadriu (Zandvliet, 1960).

El modelado preglacial (pre-Mioceno) queda representado por las superficies de bajo relieve en las cimas, siendo comunes en los Pirineos (Boissevain, 1934). Estos rellanos tienen su origen en un periodo prolongado de exposición erosiva previamente a las glaciaciones del Pleistoceno (Zandvliet, 1960; Furdada, 1988). 
Durante el Cuaternario, la acción continuada de los procesos fluvio-torrenciales ha generado una incisión en los valles hasta establecer el modelado actual. Esta red de drenaje está principalmente determinada por lineaciones tectónicas hercínicas y alpinas y por contrastes litológicos (Zandvliet, 1960). Los procesos de encajamiento de la red de drenaje, la existencia de fuertes pendientes y desniveles importantes y la presencia de discontinuidades litológicas han favorecido el desarrollo de procesos de ladera y, en consecuencia, la generación de movimientos en masa.

\section{Metodología}

La metodología general de este trabajo consiste en realizar un análisis del índice $S L$ en las zonas de $L a$ Vall d'Àssua, Romadriu y La Vall de Tor. Para llevar a cabo dicha metodología, el flujo de trabajo se ha estructurado en cinco fases (Fig. 3):

- Fase 1. Recopilación y preparación de los datos de partida.

- Fase 2. Cálculo del índice SL a partir de la herramienta Chi-Map, desarrollada y cedida para el presente estudio por José Vicente Pérez Peña, miembro del grupo de investigación del Departamento de Geodinámica de la Universidad de Granada.

- Fase 3. Postprocesado de los datos del índice SL para determinar la distribución de valores anóma- los del índice SL mediante las agrupaciones de puntos calientes (puntos con alta probabilidad de tener valores anómalamente altos del índice $\mathrm{SL}$ )

- Fase 4. Elaboración del mapa de anomalías SL.

- Fase 5. Interpretación de las anomalías del índice SL a partir de los resultados en cada cuenca.

\section{Recopilación y preparación de los datos de entrada (fase 1)}

En esta fase se han recopilado y preparado los datos necesarios para el cálculo del índice SL (fase 2). Para este cálculo se requieren cuatro archivos de entrada: (1) el Modelo Digital de Elevaciones (MDE); (2) la Acumulación de Flujo, que es un archivo ráster que contiene el número de celdas que fluyen hacia cada una de las celdas; (3) el área de drenaje de las tres cuencas de estudio y (4) los puntos de cabecera de cada una. Por otra parte, para poder elaborar la fase 5 es necesario visualizar el relieve del terreno y tener datos sobre la geología y la cartografía geomorfológica de las cuencas de estudio. Por consiguiente, se necesita un mapa de sombras del relieve, un mapa geológico y la cartografía de las cuencas de estudio. Por lo tanto, en la fase 1, se ha desarrollado una base de datos en ArcGIS 10.3.1® compuesta por los siguientes archivos que se utilizan más adelante en las fases 2 y 5 :
Fase 1

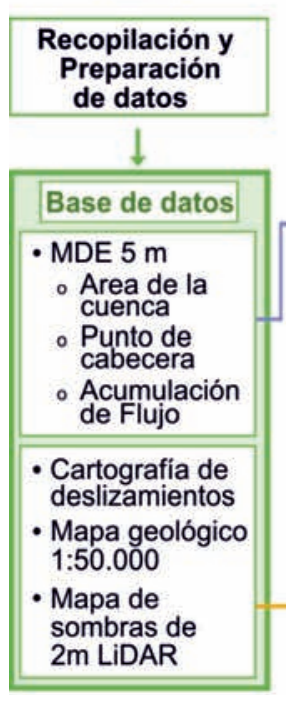

Fase 2
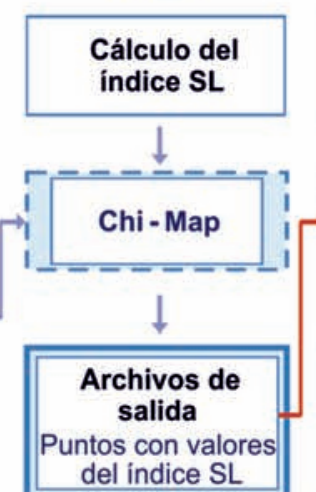

del indice SL

Fase 3
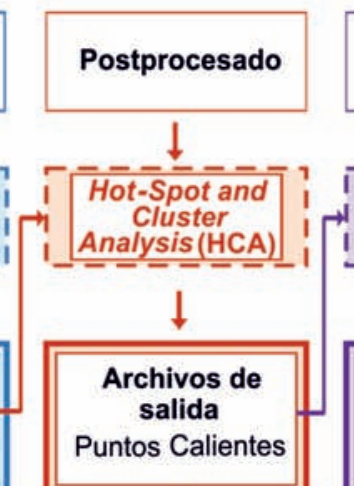

Fase 4

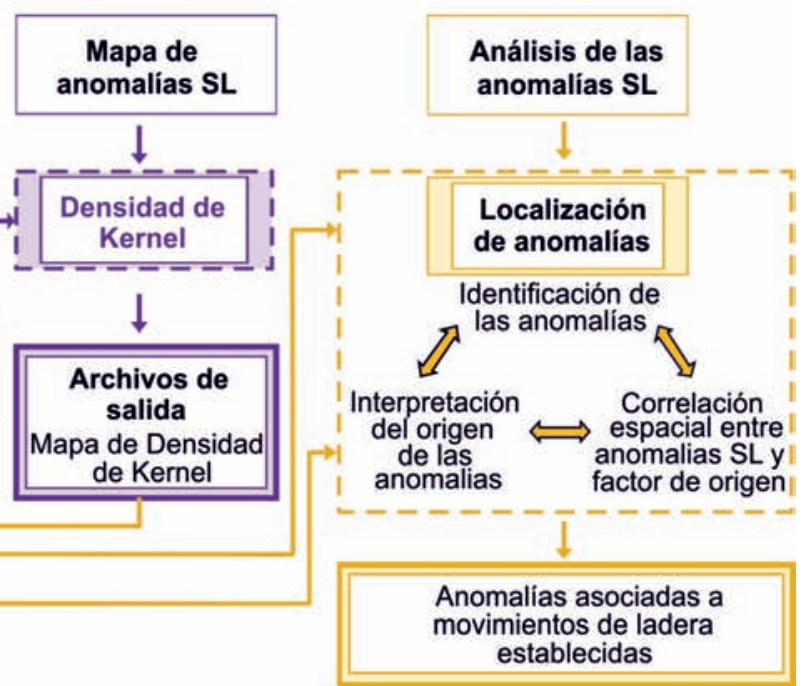

Figura 3. Esquema de las principales fases del flujo de trabajo diferenciadas por colores. Los rectángulos con bordes discontinuos son los archivos de entrada utilizados para cada fase. Mientras que los rectángulos con los bordes continuos gruesos corresponden a los resultados de cada fase.

Figure 3. Scheme of the main phases of workflow differentiated by colours. The rectangles with dashed borders correspond to the input files used for each phase, whereas the rectangles with thick borders correspond to the results of each phase. 
- EI MDE de $5 \mathrm{~m}$ realizado a partir de restitución fotogramétrica, proporcionado por el Institut Cartogràfic i Geològic de Catalunya (ICGC).

- La acumulación de flujo para cada zona. Este archivo ráster se genera a partir de la extensión de Hidrología en el paquete de herramientas de ArcGIS Spatial Analyst Tools ${ }^{\circledR}$ de la siguiente manera: En primer lugar, para reducir las imperfecciones del MDE se obtiene el relleno (fill). Después, a partir de éste, se consigue la dirección de flujo (flow direction) que muestra la dirección de drenaje de cada celda y, finalmente, la acumulación de flujo (flow accumulation), que muestra el número de celdas que fluyen hacia cada celda.

- El punto de cabecera de cada cuenca, establecido a partir de la interpretación del MDE de $5 \mathrm{~m}$.

- El área de drenaje de las cuencas, extraída automáticamente a partir del MDE de $5 \mathrm{~m}$.

- El mapa de sombras de alta resolución, generado a partir del MDE $2 \mathrm{~m}$ obtenido a partir de datos LiDAR (Light Detection And Ranging), proporcionado por el ICGC.

- La cartografía geológica de Cataluña a escala 1:50.000 facilitada por el ICGC.

- El inventario de movimientos en masa elaborado por Furdada (1988) en la cuenca de La Vall d'Àssua y por el grupo de investigación RISKNAT de la UB en la cuenca de Romadriu, parcialmente publicado en Ortuño et al. (2017).

En relación a la cartografía de movimientos de ladera, en La Vall d'Àssua se dispone un inventario de movimientos de ladera delimitados a escala 1:25.000. Por otro lado, en la cuenca del Romadriu únicamente se dispone de una cartografía detallada de escarpes asociados a inestabilidades de ladera de la zona de Portainé y no se tienen los cuerpos delimitados de inestabilidades de ladera (Ortuño et al., 2017). En algunas laderas del resto de dicha cuenca el Grupo RISKNAT ha cartografiado algunos escarpes, pero tampoco se han delimitado las áreas afectadas por movimientos en masa. Para acabar, en la base de datos creada no figura un inventario de inestabilidades de ladera en La Vall deTor, ya que, es una zona sin datos previos sobre movimientos en masa.

\section{Cálculo del índice SL con la herramienta Chi-Map (fase 2)}

Según Hack (1973), el índice del gradiente del perfil longitudinal (Stream-length gradient index, SL) se basa en detectar variaciones bruscas en la pendiente del perfil longitudinal del río provocados por una ano- malía (knickpoint). Este índice morfométrico se calcula mediante la siguiente ecuación (1) (Hack, 1973):

$$
S L=(d H / d L) * L_{t}
$$

Donde el componente $d H$ es la diferencia de altura entre los dos puntos del segmento y $d L$ es la longitud de este segmento. El valor de $L_{t}$ corresponde a la longitud acumulada desde el punto inicial, la cabecera del río, hasta el punto medio del tramo considerado donde se calcula el índice (Hack, 1973) (Fig. 4).

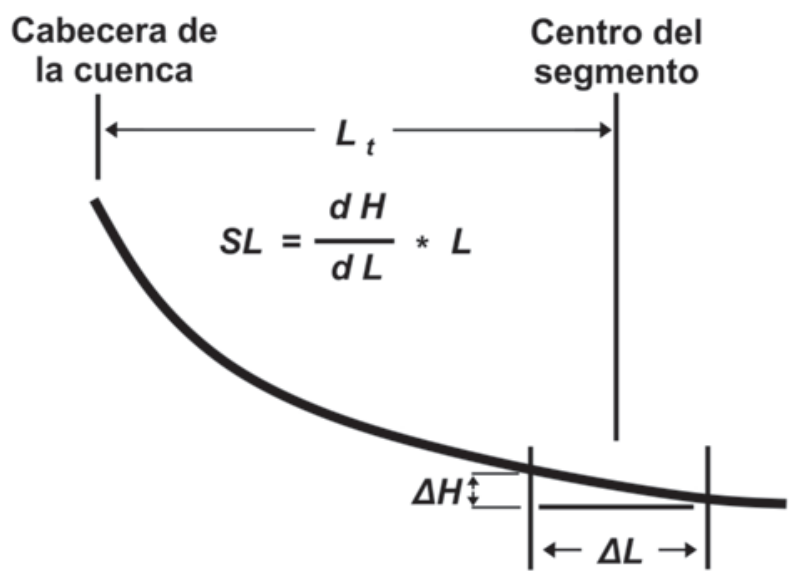

Figura 4. Ejemplo de cómo se genera el índice SL para una sección del perfil longitudinal del río. Modificado de Hack (1973).

Figure 4. Example of how the SL index is generated for a section of the longitudinal river profile. Modified from Hack (1973).

La herramienta Chi-Map permite calcular el índice $S L$ a partir de $d L$. En otras palabras, se puede modificar la longitud en la que se dividirá cada segmento del curso fluvial. Dicha herramienta presenta 5 campos de entrada que corresponden a los cuatro archivos de entrada preparados en la fase 1 y al valor de $d L$ de dicho índice. A partir del área de la cuenca y del punto de cabecera, Chi-Map establece la cabecera de la cuenca como punto de origen para procesar los datos del índice SL hasta la desembocadura del río principal. De este modo, a partir de estos indicadores y del ráster de acumulación de flujo, se crea la red de drenaje de la zona de estudio con los ríos jerarquizados desde los principales hasta los afluentes de mayor a menor categoría.

En este trabajo, se ha utilizado una longitud de segmento $d L=50 \mathrm{~m}$, ya que, Troiani et al. (2014 y 2017) obtienen valores óptimos de SL con esta longitud de segmento, utilizando un MDE de $5 \mathrm{~m}$ como el que se 
ha utilizado en este trabajo. Finalmente, una vez introducidos los cuatro archivos de entrada mencionados y el valor de $d L=50 \mathrm{~m}$, al ejecutar la herramienta se genera una entidad (shapefile) de puntos. En este archivo estos puntos están situados al centro de cada uno de los segmentos generados $y$, almacenan los datos del índice SL, la longitud, la pendiente y la altura de cada segmento (Fig. 5A).

\section{Postprocesado de los datos a partir del Análisis de Puntos Calientes (fase 3)}

Una vez obtenidos los datos del índice $S L$, para mejorar la visualización e identificación de los valores anómalos del índice $S L$ se realizó un postprocesado siguiendo la metodología propuesta por Troiani et al. (2017). Este procedimiento consiste en la aplicación
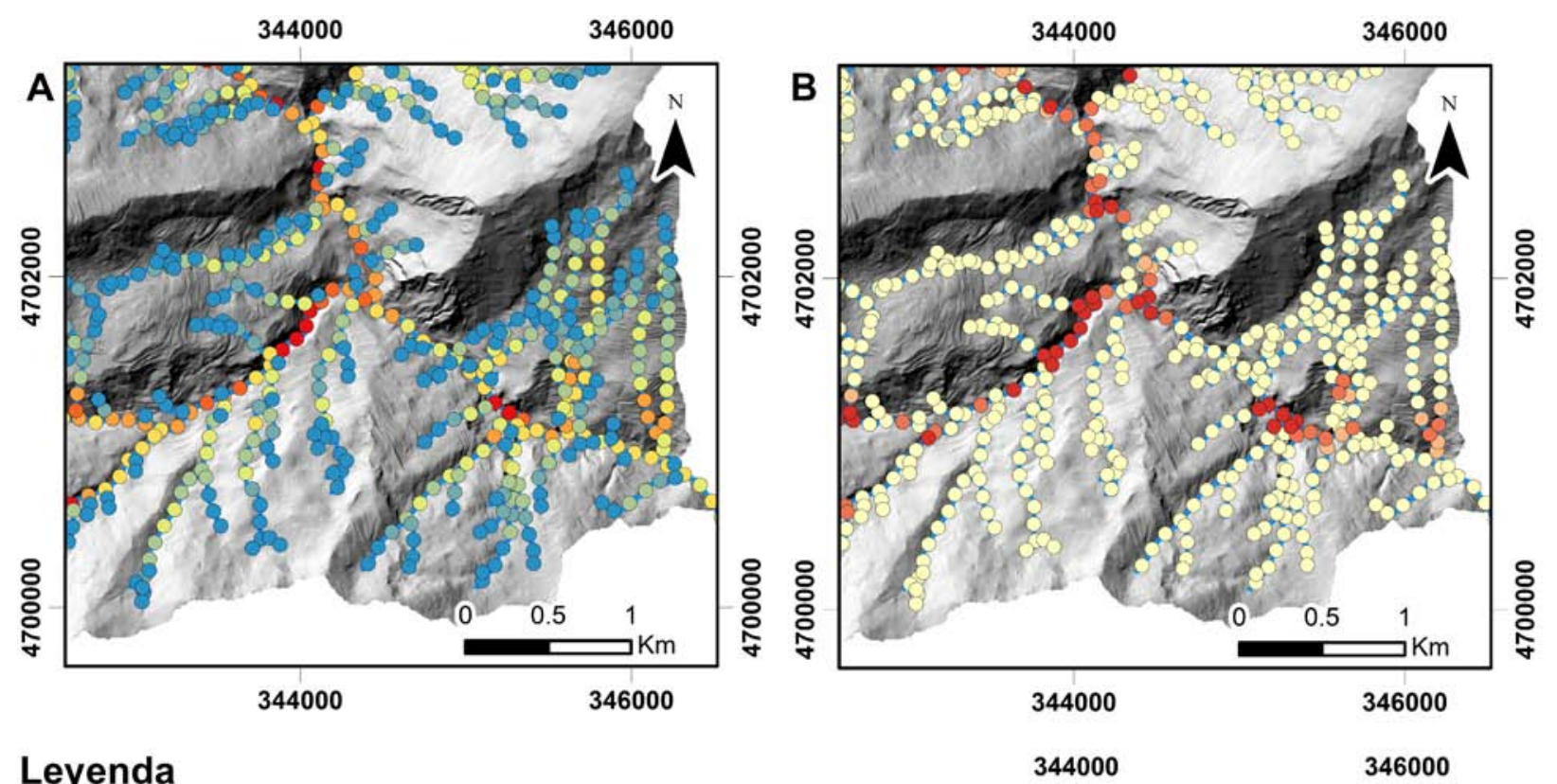

\section{Leyenda}

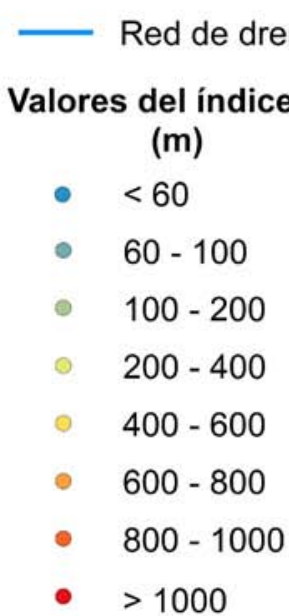

\section{Puntos Calientes Gi_Bin}

- Sin importancia

- $\quad 90 \%$ Confianza

- $95 \%$ Confianza

- $99 \%$ Confianza

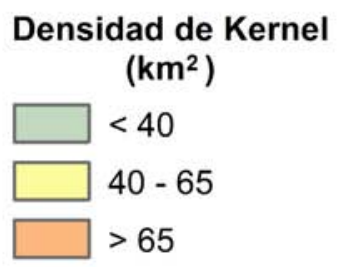

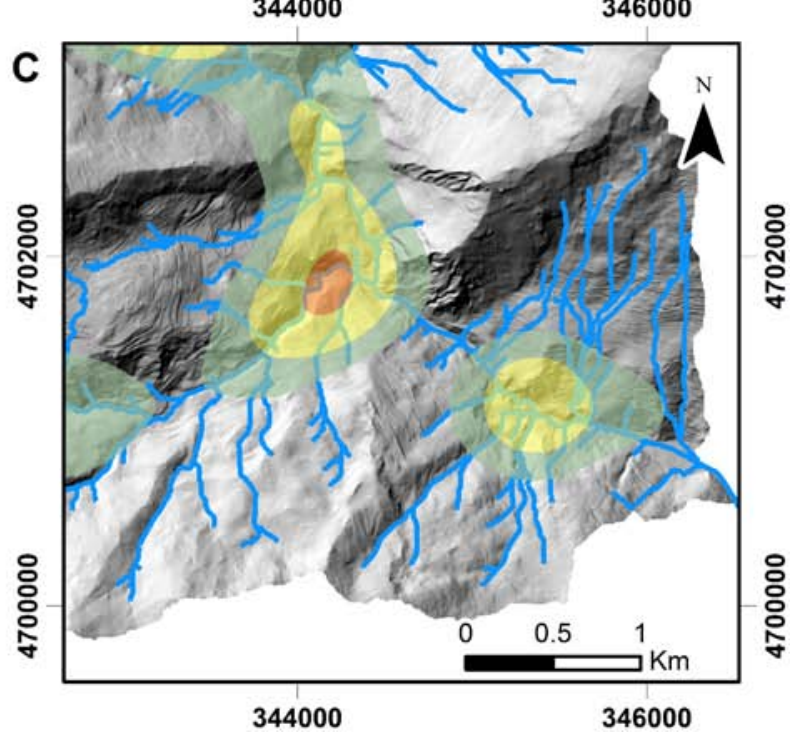

Figura 5. Fases de la obtención y tratamiento de datos. Ejemplo de los resultados obtenidos en el extremo sur oriental de la cuenca de La Vall d'Àssua: (A) Distribución de puntos de valores del índice SL a lo largo de la red de drenaje, (B) Mapa de distribución de los valores de $\mathrm{Gi}^{*}$ de puntos calientes y (C) Mapa de anomalías SL realizado por Densidad de Kernel. Mapa de sombras de la malla de $5 \mathrm{~m}$ en la subcuenca de La Vall d'Àssua utilizado de fondo y extraído del ICGC. Datum: ETRS 89, zona 31N.

Figure 5. Phases of obtaining and processing data. Example of the results obtained in the south-eastern part of La Vall d'Àssua basin. (A) Distribution of SL index values along the drainage network. (B) Map showing the distribution of Gi* values. (C) Map of SL anomalies SL carried out by Kernel Density. The background is the 5-m hillshade map of La Vall d'Àssua basin and it is extracted from ICGC. Datum: ETRS 89 , zone $31 \mathrm{~N}$. 
del Análisis de Puntos Calientes (Hotspot and Cluster Analysis, HCA) del paquete de herramientas de Estadística Espacial ${ }^{\circledR}$ de ArcGIS 10.3.1.

Este script se basa en la estadística Getis-Ord Gi* (Getis and Ord, 1992) y realiza dos cálculos. En primer lugar, el índice $\mathrm{Gi}^{*}$ determina en una distancia de búsqueda establecida (d) cual es el valor promedio de $S L$ en los puntos ubicados dentro de dicha longitud. A partir de Troiani et al. (2017), este trabajo ha tomado de referencia una distancia de búsqueda de $150 \mathrm{~m}$, ya que, utilizan este rango de valores para el mismo tamaño de malla del modelo digital del terreno. Después realiza un análisis estadístico que detecta los puntos que presentan una mayor probabilidad (puntos calientes) o una menor probabilidad (puntos fríos) de tener valores anómalamente altos del índice SL. Después de estos cálculos, esta herramienta da como archivo de salida el valor de la confianza, denominado Gi_Bin (Fig. 5B). Más allá del significado de los valores estadísticos, el HCA permite destacar aquellos tramos del río donde predominan valores altos del índice SL expresados en \% de confianza en color rojo (Fig. 5B). Además, también se pueden destacar aquellos tramos donde abundan valores bajos del índice SL. En ESRI-1 (http://desktop.arcgis.com) y Troiani et al. (2017) se encuentra una explicación más detallada de cómo funciona esta herramienta. En este proyecto, el uso de esta herramienta y la que se explica a continuación solo han servido para visualizar las anomalías principales del índice SL. En este trabajo, no se ha pretendido analizar qué valores permiten un mejor resultado en la visualización, como hicieron los autores anteriormente citados.

\section{Mapa de anomalías (fase 4)}

En la última fase del tratamiento de datos se ha realizado el mapa de anomalías, en el cual se observa la representación de superficies de contorno de la distribución de anomalías. Siguiendo la metodología de Troiani et al. (2017), para obtener este archivo ráster se ha aplicado la Densidad de Kernel (Kernel Density, $\mathrm{KD}$ ), ubicada en el paquete de herramientas de Análisis Espacial $\AA^{\circledR}$ de ArcGIS 10.3.1 ${ }^{\circledR}$.

La ecuación de kernel definida por Silverman (1986) permite zonificar la densidad de puntos calientes. Es decir, permite visualizar de forma más fácil y directa las zonas donde se concentran más datos, con mayor probabilidad de presentar valores altos del índice SL (Troiani et al., 2017) (Fig. 5C). Una descripción en detalle sobre el funcionamiento de esta ecuación y herramienta se encuentra en ESRI-2 (http://pro.arcgis.com).

\section{Análisis de las anomalías (fase 5)}

Una vez obtenidos los datos del índice SL, los puntos calientes y la distribución en polígonos de la mayor densidad de estos, se ha realizado un análisis de las anomalías encontradas en La Vall d'Àssua, el Romadriu y La Vall de Tor. Este proceso consta de tres etapas: (a) Localización de las anomalías, (b) caracterización de éstas, (c) correlación e interpretación entre las anomalías y el factor más probable que la ha originado.

\section{Localización de las anomalías del índice SL}

La ubicación de las anomalías del índice SL en La Vall $d^{\prime}$ 'Àssua y en el Romadriu se ha realizado a partir de los resultados directos del índice SL y de los puntos calientes. Mientras que, las anomalías en La Vall de Tor se han localizado a partir del resultado del mapa de anomalías de la Densidad de Kernel.

\section{Caracterización de las anomalías del índice SL}

Después de localizar dichas zonas, se ha identificado el tramo con valores anómalos del índice SL en el perfil longitudinal del valle principal de cada cuenca. Para caracterizar las anomalías del índice SL se han utilizado tres criterios en la observación de los datos directos de este índice, de los cuales, el primero y el tercero son dos procedimientos exclusivos de este trabajo:

- Media general del valor de SL.

En primer lugar, se ha observado la tendencia general del valor SL desde la cabecera hasta la desembocadura y después, se ha determinado el valor promedio de SL en Excel de todo el perfil longitudinal. De este modo, aquellos sectores que presentan un determinado valor de SL inferior o superior a la media general han permitido separar el perfil longitudinal por tramos (Fig. 6).

- División de tramos por litologías.

Troiani et al. (2014) propusieron aplicar un valor estadístico a los datos de SL, para reducir aquellas anomalías relacionadas con cambios litológicos, y poder resaltar aquellas que estuvieran ligadas a tectónica activa o inestabilidades de ladera. Aunque en este trabajo no se ha realizado un tratamiento estadístico de los datos $\mathrm{SL}$, en el perfil longitudinal se han marcado aquellas litologías que afloran en las zonas de estudio, para poder identificar aquellas anomalías producidas por cambios litológicos (Fig. 6). 
G. B. Subiela Blanco, et al., 2019. Idoneidad del índice SL para la identificación... Boletín Geológico y Minero, 130 (3): $465-487$

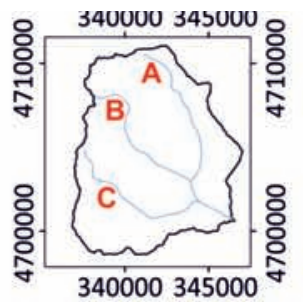

\section{Leyenda}

- Perfil longitudinal

Perfil del índice SL

Valor promedio general de SL

- - - Valor promedio de SL por tramos

\section{Anomalias}

1 Asociadas a inestabilidades de ladera

2 Asociadas a cambios litológicos

3 Asociadas a factores indeterminados

Tramo del río afectado por inestabilidades de ladera

Nuevo movimiento de ladera cartografiado

Ejemplo de movimiento de ladera asociado a anomalía

Ejemplo de movimiento de ladera no asociado a anomalía

\section{Litologias}

Dc Calizas devónicas

Dp Pizarras devónicas

Dcp Calizas y pizarras devónicas

Coaa Arcillas y areniscas cambroordovícicas
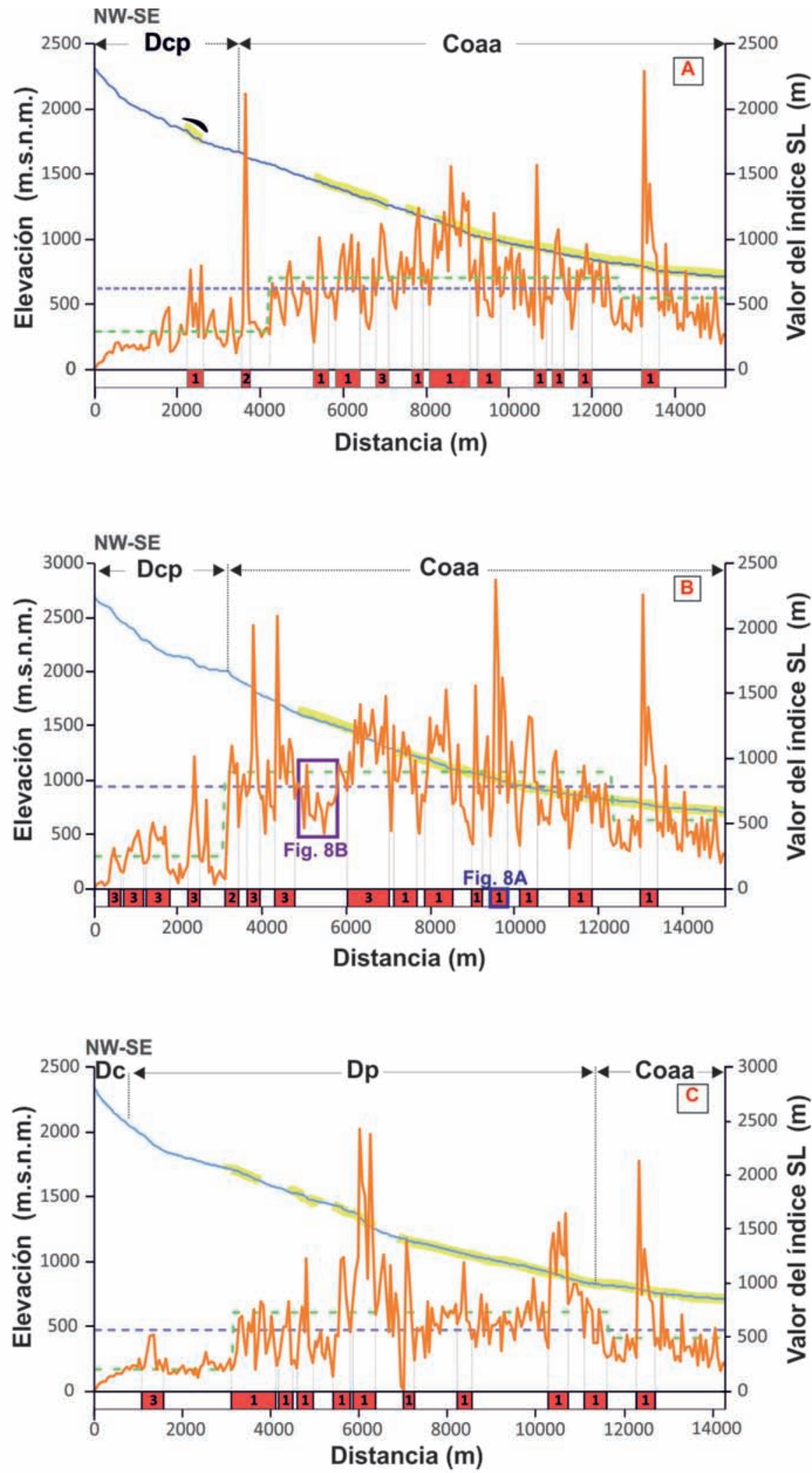

Figura 6. Perfiles longitudinales del torrente de Caregue (A), Berasti (B) y Altron (C), tendencia de los valores de SL y ubicación de las anomalías detectadas en cada torrente.

Figure 6. Longitudinal river profile of Caregue (A), Berasti (B) and Altron (C), tendency of SL values and location of the SL anomalies which are identified in each river. 
- Detección de knickpoints: salto cualitativo de los valores del índice SL

A escala local (1:20.000 aprox.), dentro del tramo caracterizado por un valor promedio SL particular, todos los picos que sobrepasaban dicho valor han sido indicativos de zonas anómalas. Para determinar la longitud aproximada de la anomalía, se han seleccionado aquellos tramos que presentan un cambio brusco de valores de SL. En otras palabras, se ha marcado como inicio de anomalía aquellas zonas que presentan un salto cualitativo desde un valor bajo de SL inferior a la media, hasta un valor alto de SL superior a la media del tramo (Fig. 6). Estas anomalías se pueden identificar en el mapa por cambios bruscos de tonalidades en los colores utilizados para representar los valores del índice SL (Fig. 5A).

\section{Correlación e interpretación entre las anomalías y el factor origen}

Con el fin de determinar la naturaleza de las anomalías y corroborar el factor origen, se ha utilizado la información de la base de datos preparada para esta fase: el mapa de sombras de $2 \mathrm{~m}$ del LiDAR, el mapa geológico y la cartografía de movimientos en masa de La Vall d'Àssua y Romadriu. Estos datos han servido para identificar si la anomalía ha sido producida por un cambio litológico, inestabilidad de ladera $u$ otros factores. La visualización de estos 3 archivos en Google Earth, junto con los valores directos de SL, agrupaciones de valores altos de SL (puntos calientes) y de Densidad de Kernel, ha facilitado la identificación del factor que puede estar produciendo la anomalía.

Para determinar el factor que origina alguna de las anomalías detectadas es necesario conocer el contexto geológico y geomorfológico de las zonas de estudio. Así, la actual actividad tectónica en los Pirineos catalanes es relativamente moderada (Goula et al. 1999; Lacan and Ortuño, 2012). Por lo tanto, en principio se ha considerado que la probabilidad de encontrar anomalías asociadas a una falla activa es baja o nula. Los dos factores que pueden generar anomalías con mayor probabilidad en las zonas estudiadas en este trabajo son principalmente los cambios litológicos y los movimientos en masa. Una anomalía producida por un cambio litológico se podrá identificar si coincide espacialmente con un contacto litológico en el mapa geológico, o bien, se verán relieves distintos producidos por diferente resistencia a la erosión en el mapa de sombras. Por otro lado, se podrá indicar una anomalía asociada a un deslizamiento, siempre y cuando, muestre evidencias tales como morfologías asociadas a movimientos de ladera $\mathrm{u}$ otros indicadores como, por ejemplo, obstrucción del canal principal por el alcance de la masa deslizada hasta al fondo de valle.

\section{Resultados}

En esta sección se exponen los resultados de la identificación de las anomalías del índice SL en el fondo de valle en las cuencas de La Vall d'Àssua, Romadriu y La Vall de Tor. Se han seleccionado varios ejemplos claros en la relación entre la anomalía y el efecto producido por el deslizamiento. Por otra parte, también se mostrará un caso discutible para demostrar la complejidad de dicha asociación.

\section{Anomalías del índice SL en La Vall d'Àssua}

En La Vall d'Àssua, el valor promedio del índice SL es de 561 m en Caregue (Fig. 6A), 785 m en Berasti Fig. $6 B)$ y $564 \mathrm{~m}$ en Altron (Fig. 6C). La mayor concentración de anomalías se distribuye en la parte central de los torrentes, dentro de las arcillas y areniscas de edad Cambro-Ordovícica, en estas zonas, el índice SL presenta un valor promedio de $706 \mathrm{~m}$ en Caregue (Fig. 6A), $901 \mathrm{~m}$ en Berasti (Fig. 6B) y $726 \mathrm{~m}$ en Altron (Fig. 7C) superior a la media general. Además, estas anomalías reflejan importantes saltos cualitativos entre valores bajos y altos de SL.

En esta cuenca, se han detectado 45 anomalías (Fig. 7), de las cuales un $55,7 \%$ pueden asociarse a inestabilidades de ladera, un $39,9 \%$ a factores indeterminados y un $4,7 \%$ se producen por cambios litológicos. Las anomalías asociadas a movimientos de ladera se correlacionan con 28 movimientos en masa presentes entre los 32 movimientos de ladera cartografiados por Furdada (1988) y han permitido identificar 2 nuevas inestabilidades de ladera no identificadas con anterioridad. El flujo de tierras del Puente de Caregue en el torrente de Berasti, caracterizado por Corominas and Alonso (1984) y después por Furdada (1988), será el ejemplo representante de los 28 movimientos de ladera para comentar la casuística del origen de la anomalía por la presencia de un movimiento de ladera (Fig. 8A).

Entre los 32 fenómenos cartografiados, el índice SL no detecta 3 cuerpos deslizados que se ubican cerca de las cabeceras de los torrentes, y por ende no generan anomalía en el perfil longitudinal del río. Por lo tanto, el índice SL no resalta knickpoints cuando la distancia es pequeña desde el centro del segmento 


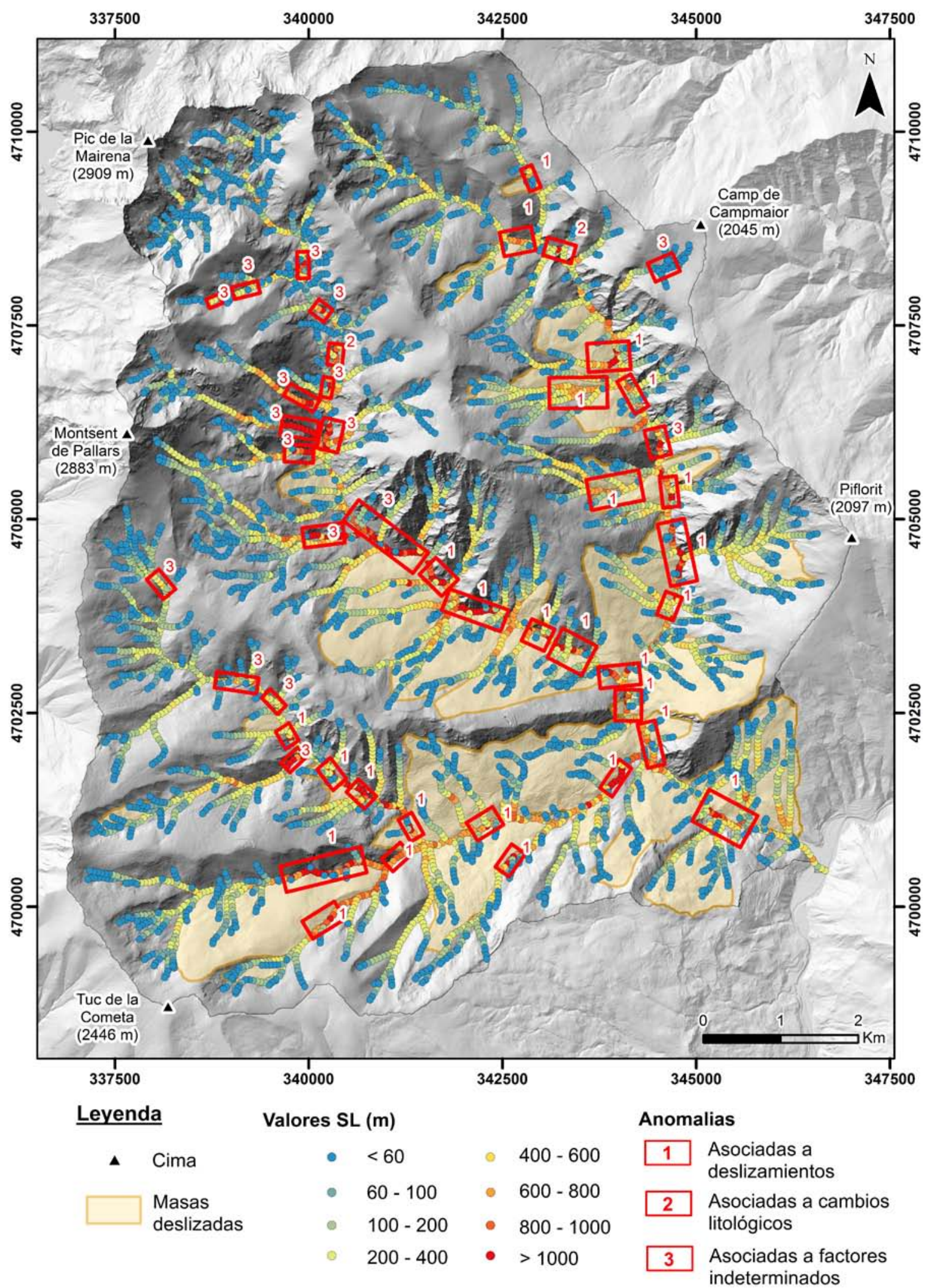

Figura 7. Localización de las 45 anomalías de SL identificadas en La Vall d'Àssua. Datum: ETRS 89, zona 31 N. El mapa de sombras de 2 m LiDAR es utilizado de fondo.

Figure 7. Localization of 45 SL anomalies which are identified in La Vall d'Àssua basin. Datum: ETRS 89, zone 31 N. The background is a 2 m LiDAR hillshade map. 
analizado respecto a la cabera. Este rasgo es una limitación del método del índice SL que se desarrolla con más detalle en el apartado de discusiones. Además, tampoco se ha identificado por el índice SL el desli- zamiento de escala menor del complejo de Bordes de Llesui, localizado en el torrente de Berasti, el cual será utilizado de ejemplo de un deslizamiento no asociado a anomalías del índice SL en La Vall d'Àssua (Fig. 8B).
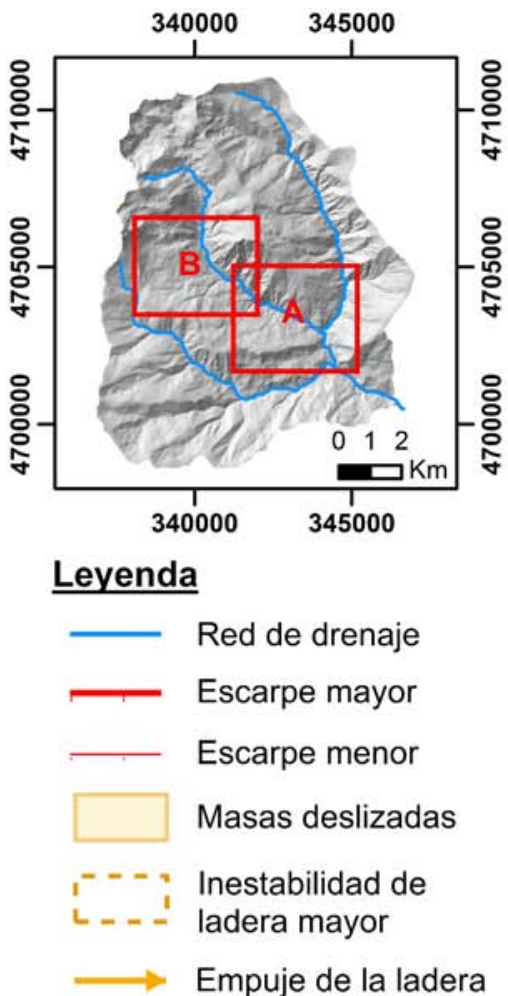

Valores SL ( $m)$

$$
\begin{array}{ll}
\quad & 200-400 \\
& 400-600 \\
- & 600-800 \\
-\quad & 800-1000 \\
-\quad>1000
\end{array}
$$

\section{Anomalias}

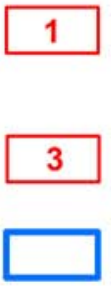

Asociadas a movimientos de ladera

Asociadas a factores indeterminados

Asociada al Puente de Caregue
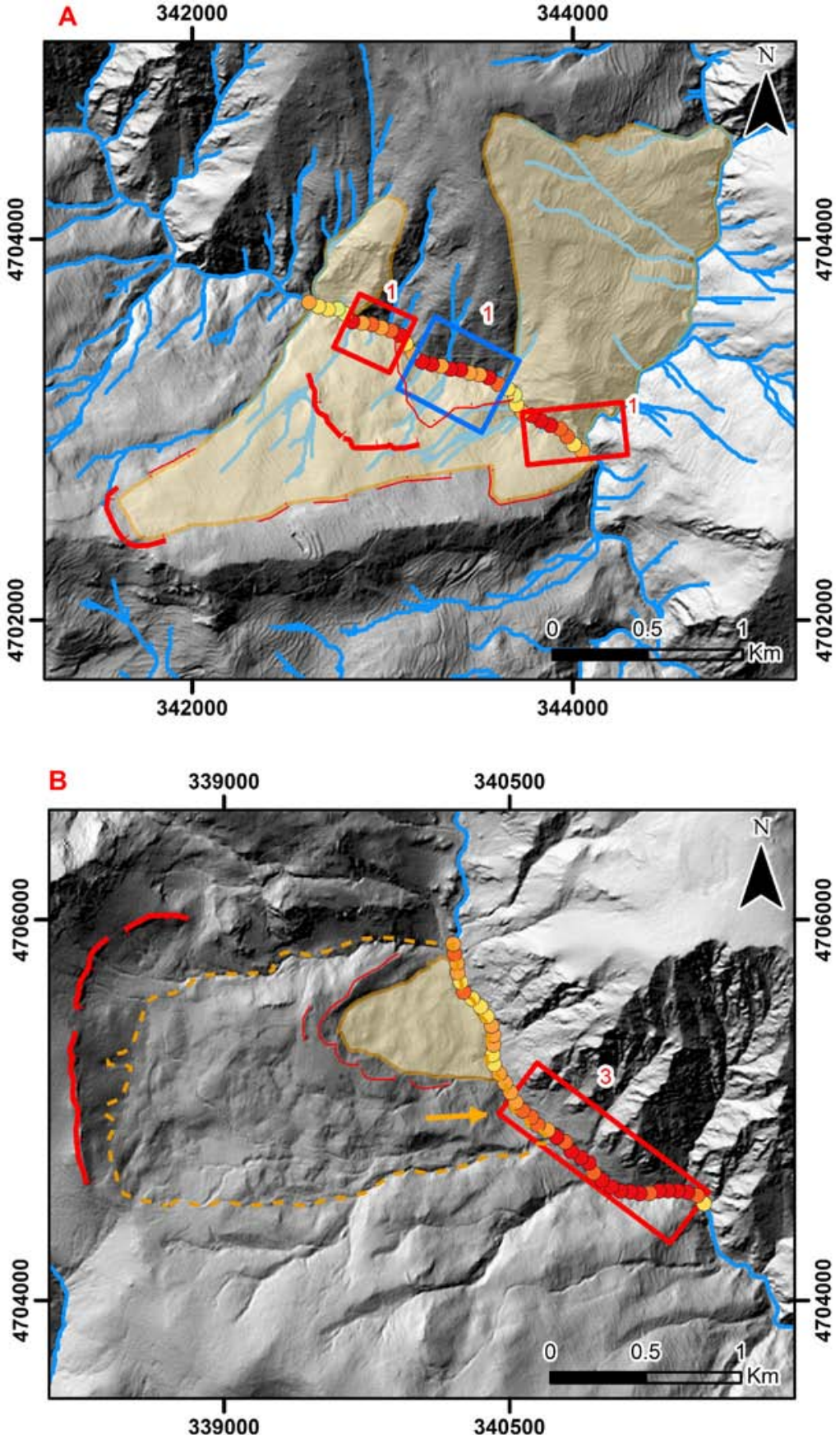

Figura 8. A) Anomalía asociada al movimiento del terreno de la ladera sur que destruyó el puente de Caregue en noviembre de 1982. B) Reactivación del frente de un deslizamiento mayor, pero no detectado por valores altos del índice SL. En estas figuras solo se muestran los valores y anomalías del índice SL de estos tramos del río.

Figure 8. A) An anomaly associated with south-western mass movement, which destroyed the Caregue bridge in November, 1982. B) Partial reactivation of the front of a larger mass movement which it is not detected by high values of the SL index. In this figure only the values and anomalies of the SL index of both section of the river are shown. 
En resumen, ahora la cartografía de La Vall d'Àssua presenta 34 inestabilidades de ladera con los dos nuevos movimientos en masa, de 0,06 y de $2,35 \mathrm{~km}^{2}$ identificados gracias al índice $\mathrm{SL}$, el cual este último se presenta en el próximo apartado (Fig. 8B).

\section{Flujo de tierras del Puente de Caregue (torrente de Berasti)}

En general, el rio Berasti presenta unos altos valores del índice SL en el sector del valle donde están los movimientos complejos de ladera descritos por Furdada (1988) (Fig. 8A). En este caso, antes de la confluencia con el torrente de Caregue, el pico máximo de SL se localiza en el lugar donde este deslizamiento rotacional destruyó el puente de Caregue en el temporal de lluvias torrenciales en noviembre de 1982 (Fig. 6B). Actualmente, esta inestabilidad de ladera está considerada activa/intermitente en la base de datos de movimientos en masa de Cataluña (Corominas and Alonso, 1984; LLISCAT, http://siurana.icgc.cat/lliscat/).

\section{Complejo de Bordes de Llesui (torrente de Berasti)}

Este movimiento de ladera, de un área de $0,24 \mathrm{~km}^{2} \mathrm{y}$ caracterizado por un componente rotacional en cabecera y flujo en el pie (Furdada, 1988), no tiene anomalías asociadas (Fig. 8B). En el perfil longitudinal de Berasti, los datos de SL son inferiores a la media de SL (901 m) en el tramo central (Fig. 8B). Sin embargo, esta ladera forma parte de una masa deslizada mayor. Aguas abajo del movimiento en masa menor hay una anomalía (Fig 9B). Probablemente, el tramo de la anomalía más cercano a la inestabilidad de ladera mayor estará siendo afectado por el empuje de éste. Sin embargo, se clasifica como indeterminada, ya que, la larga longitud del tramo anómalo sugiere que hay otro factor que no se ha podido determinar que está provocando la variación en la pendiente del río aguas abajo. Este caso es un ejemplo de reactivación del frente de un deslizamiento mayor $\left(2,35 \mathrm{~km}^{2}\right)$, donde el encajamiento de la red de drenaje produce la erosión del frente de dicho deslizamiento, provoca la desestabilización de esta ladera y genera un deslizamiento de menor escala $\left(0,24 \mathrm{~km}^{2}\right)$.

\section{Anomalías del índice SL en el Romadriu}

A grandes rasgos, la cuenca del Romadriu se diferencia de La Vall d'Àssua por el perfil longitudinal, el cual presenta una morfología convexa (Fig. 9), y por la configuración de las anomalías, ya que en este caso se localizan mayoritariamente en el tramo final del río, a partir de los $17,9 \mathrm{~km}$ de distancia a la cabecera (Fig. 9), a diferencia de La Vall d'Àssua donde se ubican en el tramo central de los torrentes de Caregue, Berasti y Altron. El valor promedio general de SL del río Romadriu es de $613 \mathrm{~m}$, presentando primero en la zona de anomalías un valor de $980 \mathrm{~m}$ y después, a partir de 25,5 km de distancia desde la cabecera, un valor de $2451 \mathrm{~m}$ (Fig. 9). Estos saltos en los valores de SL se observan claramente en el perfil longitudinal del Romadriu (Fig. 9). Además, se ha detectado que estas anomalías principales también se ubican dentro de la unidad de arcillas y areniscas de edad CambroOrdovícica.

En toda la cuenca, se han identificado 20 tramos anómalos. Un $40 \%$ pueden estar asociados a movimientos en masa, un $25 \%$ a contactos litoestructurales, un $25 \%$ pueden ser por la interacción de varios factores o factores indeterminados y un $10 \%$ a presas artificiales (Fig. 10). Los tres ejemplos de anomalías seleccionados se localizan a lo largo del río Romadriu (Fig. 10) y, en principio, dichas anomalías no pueden ser producidas por cambios litológicos porque se ubican en la unidad de arcillas y areniscas cambro-ordovícicas. En este sector, aunque las variaciones en los valores de SL pueden ser producidas por un único factor, la interacción de otros factores indeterminados no se debe descartar.

Como se ha mencionado anteriormente, la cartografía de movimientos en masa del Romadriu se caracteriza por un inventario de escarpes de inestabilidades de ladera realizado por RISKNAT, pero sin Ilegar a delimitar el área afectada por estos fenómenos. A partir del mapa de sombras de 2 m LiDAR, la ubicación de los escarpes inventariados y de las anomalías del índice SL se ha delimitado 2 movimientos en masa con dimensiones de $0,26 \mathrm{~km}^{2}$ (Fig. 11A) y 0,47 $\mathrm{km}^{2}$ (Fig. 11B), ubicados cerca del tramo final del fondo de valle, que no habían sido previamente cartografiados.

El movimiento de ladera mostrado en la Fig. 11A, ubicado en la ladera suroeste y que presenta un aspecto irregular con varios rellanos, ha sido localizado gracias a las anomalías del índice SL. Este knickpoint también puede ser formado por la erosión del frente y la consecuente desestabilización de la vertiente.

El deslizamiento mostrado en la Fig. 11B, delimitado a partir del inventario de escarpes de RISKNAT y de una anomalía del índice SL, se ubica aguas abajo de una de las cuatro presas del Romadriu. Este knickpoint puede indicar que el talud se está desestabili- 


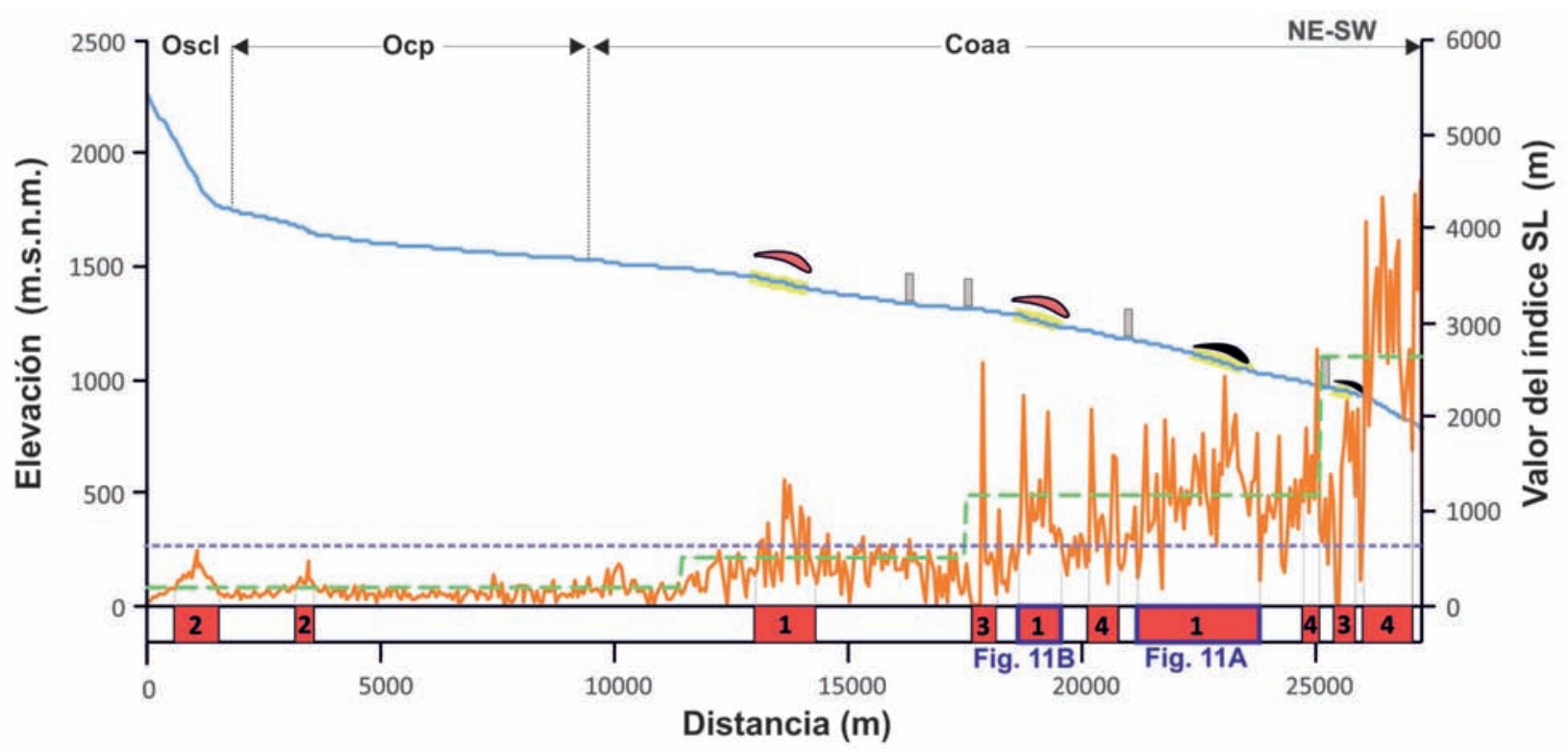

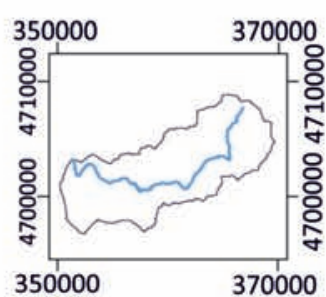

Leyenda

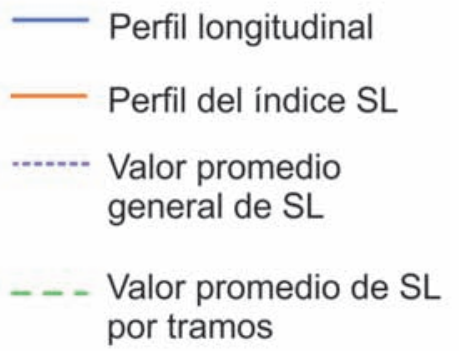

\section{Anomalías}

1 Asociadas a inestabilidades de ladera

2 Asociada a cambios litológicos

3 Asociada a factores indeterminados

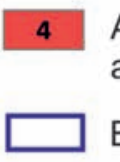

Asociada a presas antrópicas

Ejemplo de anomalia

Tramo del río afectado por inestabilidades de ladera
Inestabilidad de ladera delimitada

Nueva inestabilidad de ladera cartografiada

Presas antrópicas

\section{Litologias}

Ocp Calizas y pizarras ordovícicas

Oscl Calizas ordovícicas y lutitas silúricas

Coaa Arcillas y areniscas cambroordovícicas

Figura 9. Perfil longitudinal del río Romadriu, distribución de las anomalías en este torrente y ubicación de las anomalías. Figure 9. Longitudinal river profile of Romadriu and distribution and location of SL anomalies.

zando, empujando el río y modificando la pendiente de ese tramo. Esta desestabilización puede ser producida por la erosión fluvial en épocas de lluvias intensas o en desembalses repentinos de dicha presa.

\section{Anomalías del índice SL en La Vall de Tor}

Las anomalías de La Vall de Tor se distribuyen principalmente por el valle del río en Noguera de Tor y el Barranco de Boixedo (Fig. 12). En general, los valores de SL son de $446 \mathrm{~m}$ en Boixedo (Fig. 13B) y de $466 \mathrm{~m}$
Noguera de Tor (Fig. 13A). En las zonas centrales de ambos ríos se ubican las anomalías de mayor intensidad, con una distribución parecida a la de La Vall d'Àssua. También coincide en que estos knickpoints se ubican en la misma unidad cambro-ordovícica de La Vall d'Àssua y Romadriu.

En esta cuenca se han identificado 30 tramos con valores anómalos de $\mathrm{SL}$, de los cuales un 33\% pueden estar asociados a movimientos en masa, un $23 \%$ a contactos litoestructurales y un $44 \%$ pueden ser ocasionados por la interacción de varios factores indeterminados (Fig. 12). Del $33 \%$ de anomalías del 


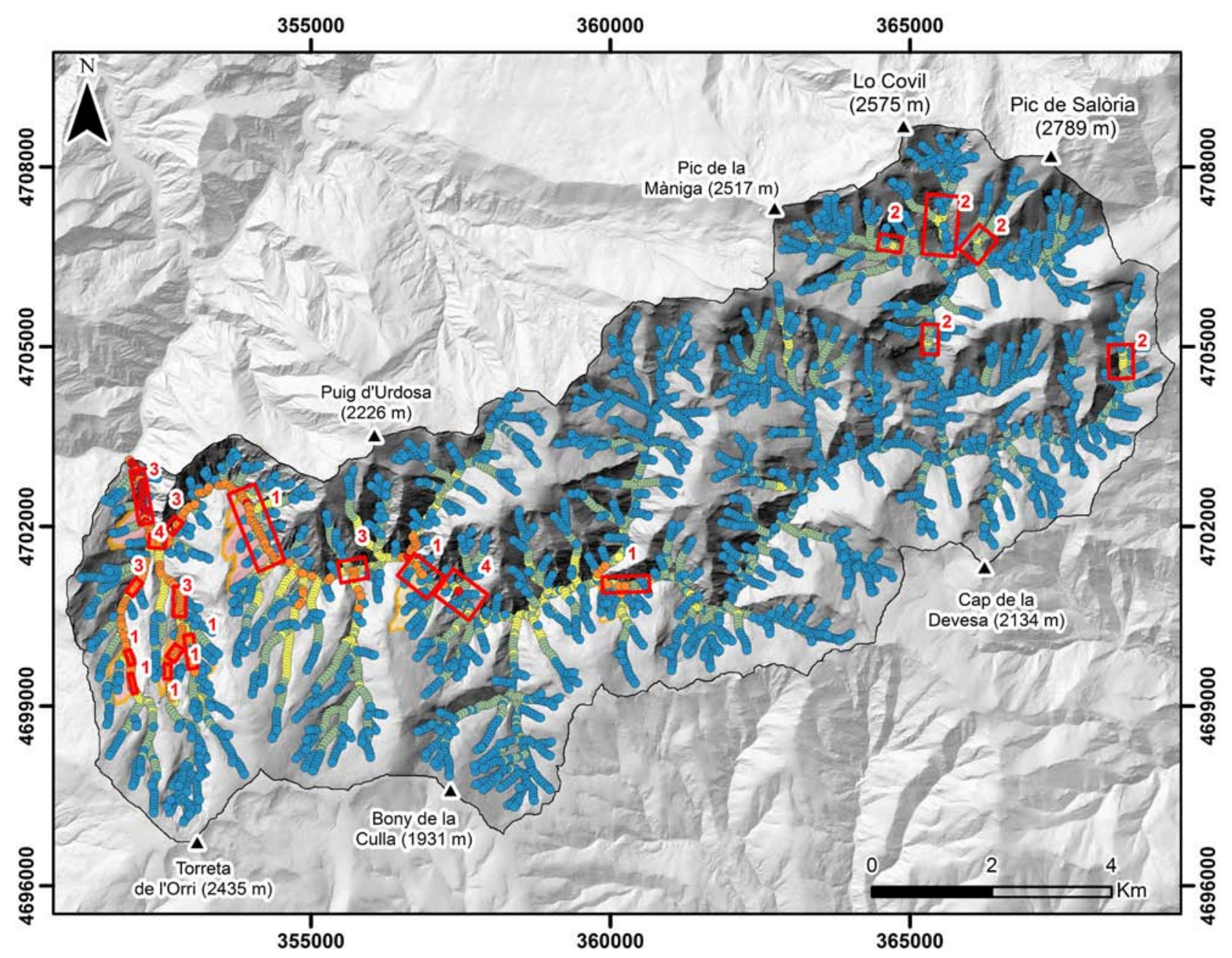

\section{Leyenda}

Cima

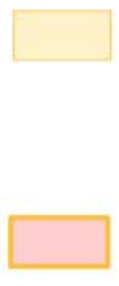

Cuerpos delimitados a partir de los escarpes y de las anomalías del indice SL

Cuerpos deslizados de nuevos deslizamientos identificados
Valores del índice SL $(\mathrm{m})$
- $\quad<155.66$

- $\quad 155.66-450.90$

- $\quad 450.90-999.94$

- $999.94-2237.12$

- $\quad>2237.12$
Anomalias

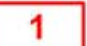

2
Asociadas a inestabilidades de ladera

Asociadas a cambios litológicos

3 Asociadas a factores indeterminados

4 Asociadas a presas antrópicas

Figura 10. Localización de las 20 anomalías identificadas en la cuenca del Romadriu. Datum: ETRS 89, zone 31 N. Fondo: mapa de sombras de 2 m LiDAR.

Figure 10. Location of 20 SL anomalies identified in Romadriu basin. Datum: ETRS 89, zone 31 N. A 2 m LiDAR hillshade map is used as a background.

índice SL ligadas a inestabilidades de ladera, se han podido identificar 7 movimientos en masa de dimensiones entre $0,03 \mathrm{~km}^{2}$ y $3,08 \mathrm{~km}^{2}$ que alcanzan el fondo de valle. A continuación, se mostrará el caso ejemplar de las anomalías relacionadas con el movimiento de ladera de Boixedo (Fig. 14).

El valle de Boixedo presenta 13 anomalías (Fig. 14), la mayoría de las cuales estarían asociadas a una 

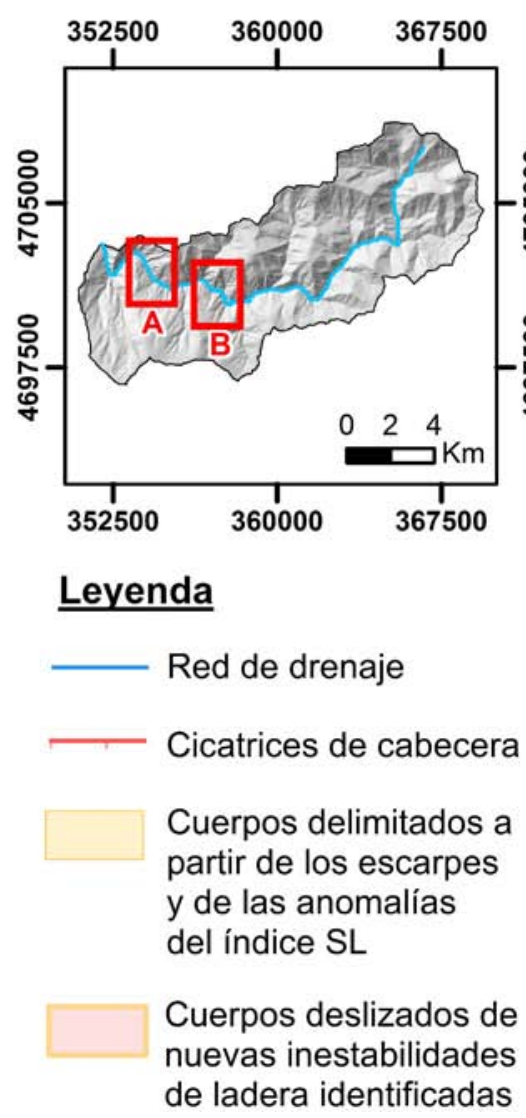

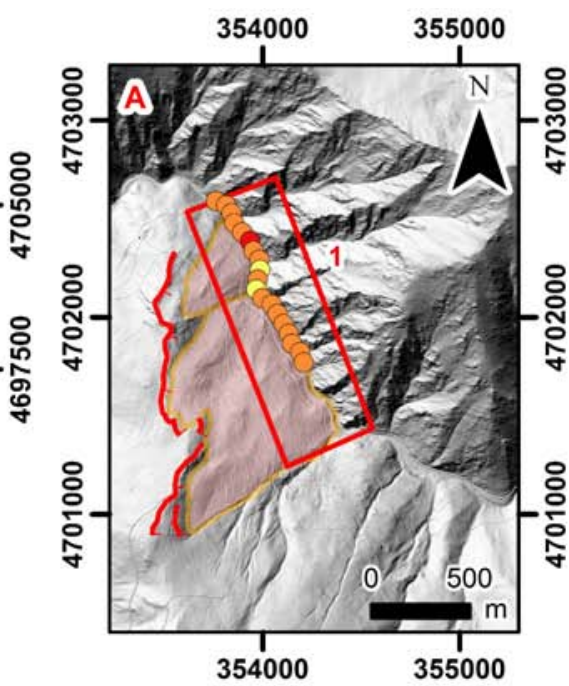

Valores del índice SL (m)

$<156$

$156-451$

$451-1000$

$1000-2237$

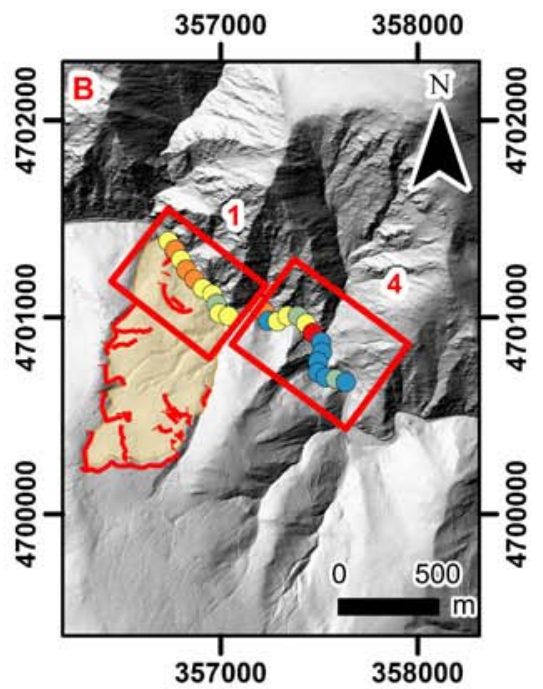

$>2237$

Anomalias

1 Zonas asociadas a inestabilidades de ladera

4

Zonas asociadas a presas antrópicas

Figura 11. Ejemplos de anomalías del índice SL asociadas a movimientos en masa. A) Identificación de una nueva inestabilidad de ladera en la cuenca del Romadriu a partir de las anomalías del índice SL. B) Deslizamiento, cerca del embalse del Romadriu (4), delimitado por los escarpes y por la anomalía del índice SL.

Figure 11. Examples of SL anomalies associated with landslides. A) Identification of a new slope failure in Romadriu basin owing to location of SL anomalies. B) Landslides near Romadriu antropic dam (4). This mass movement is delimited by scarps and an SL anomaly.

gran inestabilidad de ladera que afecta a la ladera suroeste del valle. Al realizar una vista general de este valle, en la ladera citada se reconocen morfologías asociadas a cuerpos movilizados como escarpes, rellanos y formas lobulares que ocupan una superficie de $3 \mathrm{~km}^{2}$ (Fig. 14). El mapa geológico 1:50 000 podría indicar evidencias de los procesos de inestabilidad, ya que, en las cabeceras se indica la presencia de depósitos coluviales cuaternarios.

Los depósitos de estos movimientos en masa alcanzan el fondo de valle del torrente de Boixedo, aunque se encuentran ya erosionados por el río (Fig. 14). Posiblemente la erosión fluvial está provocando la desestabilización de la ladera y, en consecuencia, el empuje de dicho fenómeno esté modificando la pendiente en algún tramo del torrente. La anomalía principal sería el mayor pico del índice SL en el gráfico del perfil longitudinal de Boixedo (Fig. 13), que se ubica después de una ladera erosionada. En este caso, la erosión de la ladera puede estar provocando la gene- ración de un movimiento en masa menor dentro de la inestabilidad de ladera de Boixedo (Fig. 14).

\section{Discusiones}

En este trabajo, la utilización del índice SL ha permitido determinar algunas limitaciones sobre su cálculo, la identificación de anomalías y la interpretación de éstas.

\section{Limitaciones del método del índice SL}

Como indica la fórmula de Hack (1973), el índice SL es una multiplicación de la longitud total desde la cabecera hasta el punto analizado por el gradiente de un tramo de una longitud determinada. Esto significa que el parámetro de la longitud es un factor importante que condicionará el valor de SL. Cuanto más 


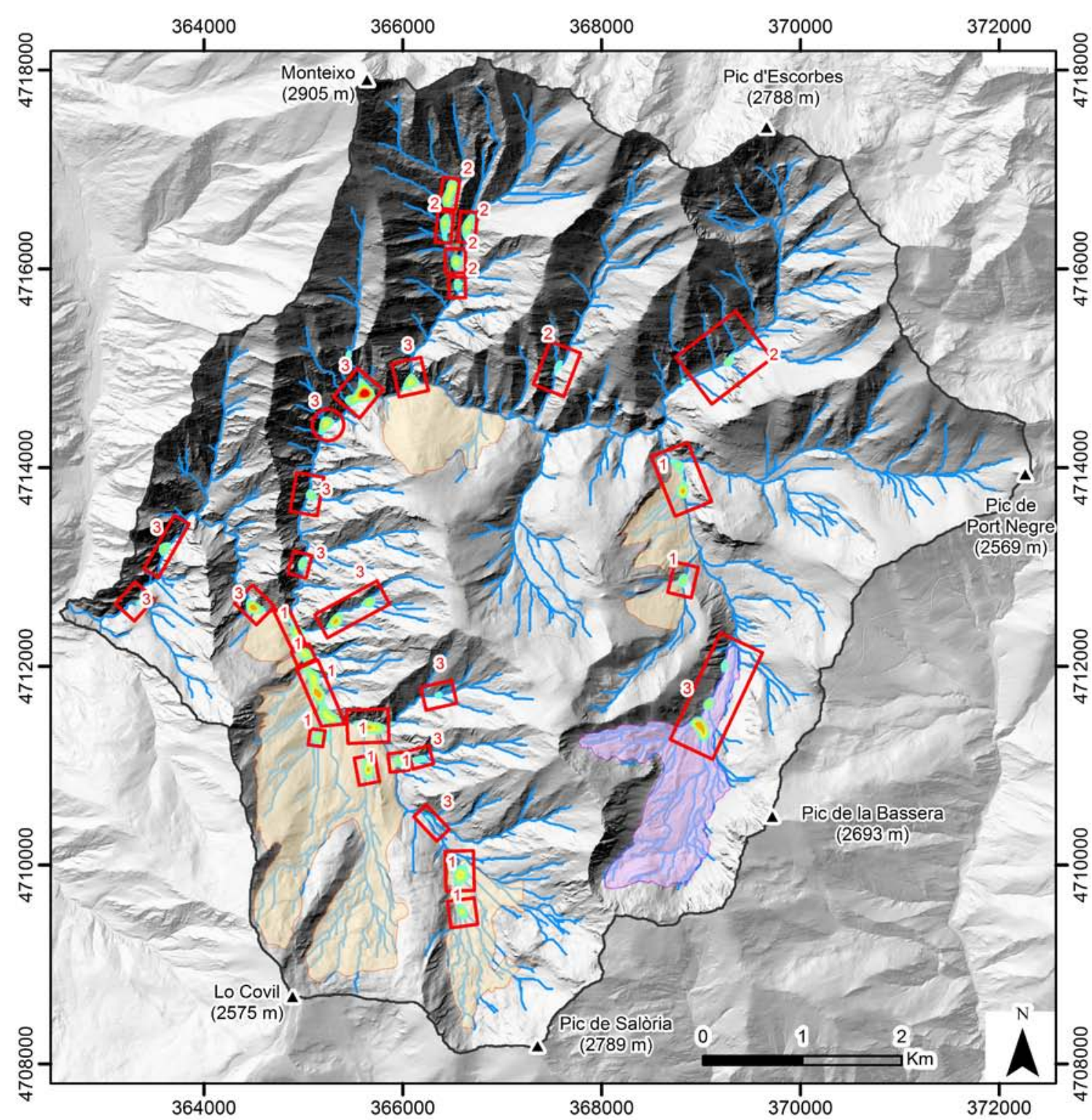

Leyenda

- Cima

Red de drenaje

Modelado periglacial

$\square$ Depósitos periglaciales

\section{Modelado de ladera}

Cuerpos deslizados asociados a anomalias
Cuerpos deslizados no asociados a anomalias

\section{Densidad de Kernel $\left(\mathrm{Km}^{2}\right)$}

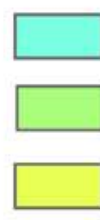

$500-600$

$600-700$

$700-800$

$800-900$

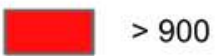

\section{Anomalias}

1 Zonas asociadas a inestabilidades de ladera

2 Zonas asociadas a cambios litológicos

3 Zonas asociadas a factores indeterminados

Figura 12. Localización de las 24 anomalías de SL identificadas en La Vall de Tor. Datum: ETRS 89, zone 31 N. La imagen de fondo es el mapa de sombras de $2 \mathrm{~m}$ LiDAR, disponible en www.icgc.cat.

Figure 12. Location of 24 SL anomalies of La Vall de Tor basin. Datum: ETRS 89, zone31 N. The background is a 2 m LiDAR hillshade map, which is available at www.icgc.cat. 

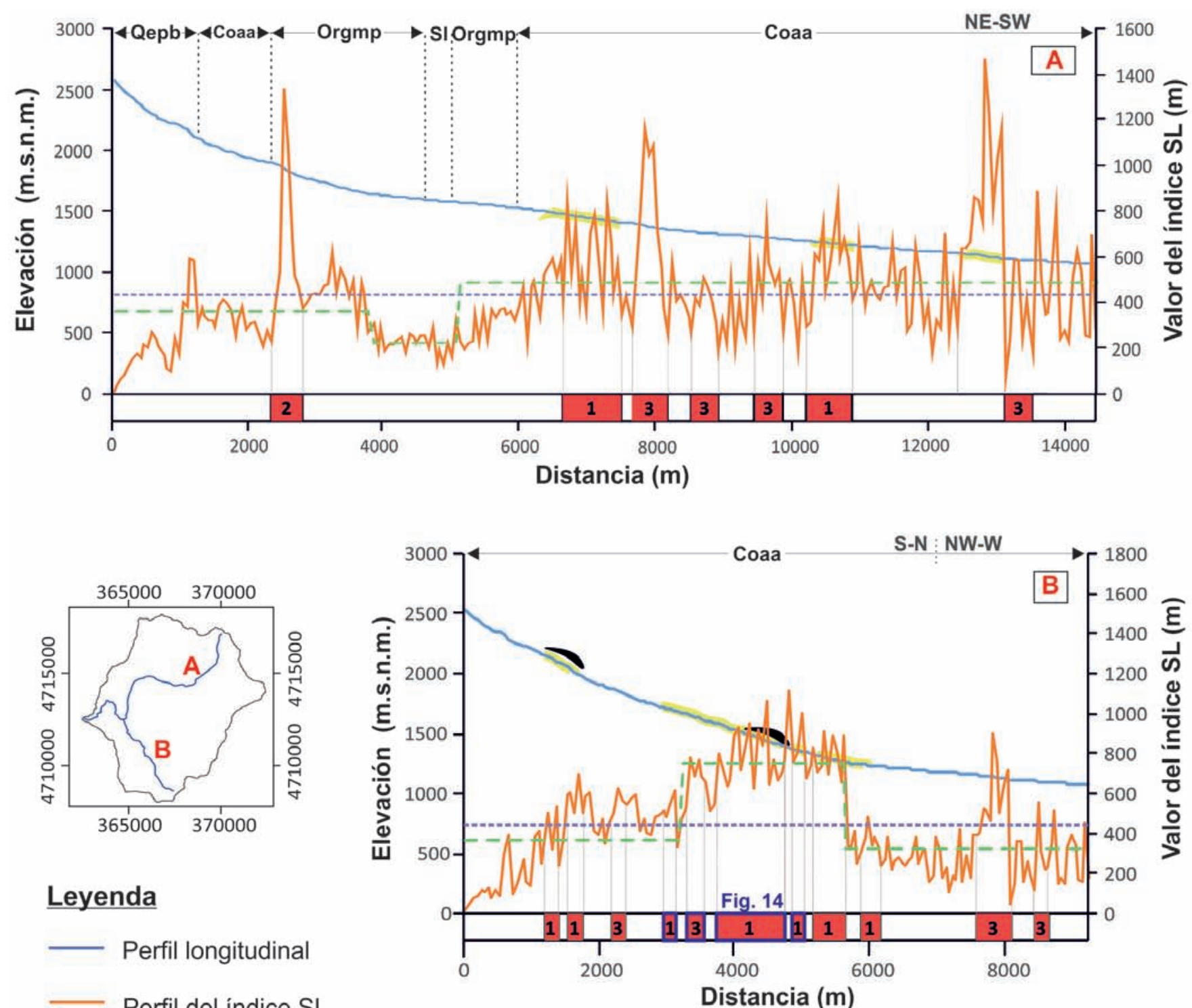

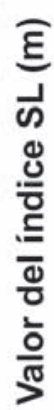

\section{Leyenda}

Perfil longitudinal

— Perfil del índice SL

\section{...-.. Valor promedio} general de SL Valor promedio de
SL por tramos

\section{Anomalías}

1 Asociadas a inestabilidades de ladera

2 Asociadas a cambios litológicos
3 Asociadas a factores indeterminados

Tramo del rio afectado por inestabilidades de ladera
- Nueva inestabilidad de ladera cartografiada

Ejemplo de anomalia
Litologias

Qepb Depósito de chancales cuaternarios

SI Lutitas silúricas

Orgmp Areniscas, microconglomerados y pizarras del Ordovícico

Coaa Arcillas y areniscas cambroordovícicas

Figura 13. Perfil longitudinal de Noguera de Tor (A) y el torrente de Boixedo (B), valores del índice SL y ubicación de las anomalías de SL. Figure 13. Longitudinal river profile of Noguera de Tor (A) and Boixedo (B), SL values and location of SL anomalies. 


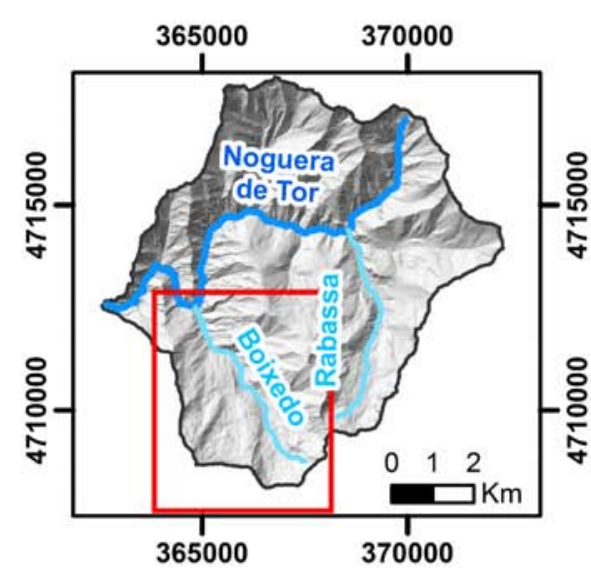

\section{Leyenda}

- Cima

\section{Red de drenaje \\ Escarpes menores}

Cuerpos deslizados asociados a anomalías

\section{Densidad de Kernel $\left(\mathrm{Km}^{2}\right)$}
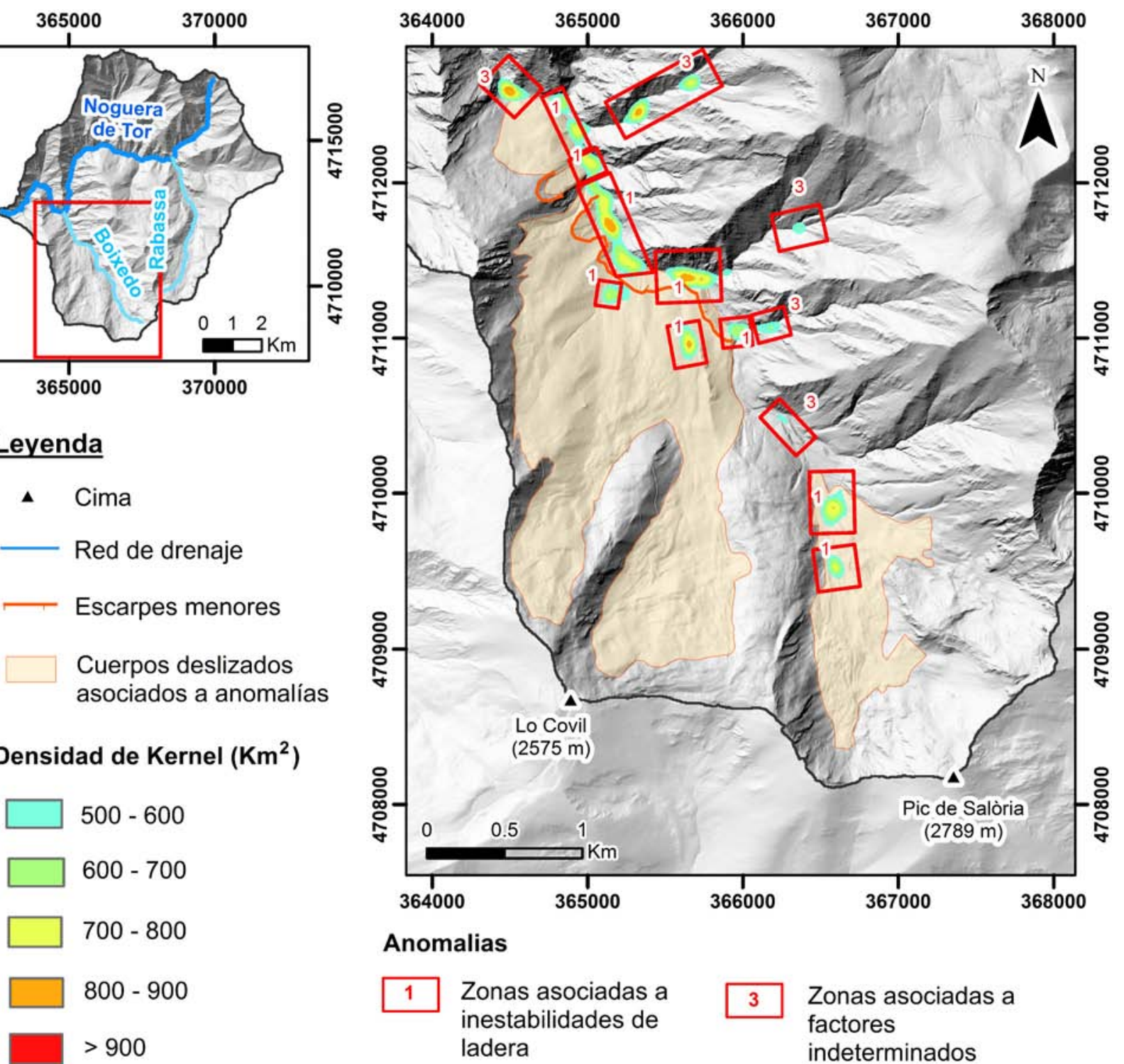

\section{Anomalias}
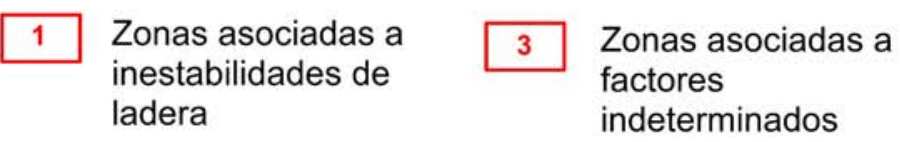

Figura 14. Localización de las anomalías asociadas a los movimientos en masa del valle de Boixedo. Datum: ETRS 89, zona 31 N. Fondo de imagen: mapa de sombras de 2 m LiDAR, disponible en www.icgc.cat.

Figure 14. Location of SL anomalies associated with mass movements in Boixedo basin. Datum: ETRS 89, zone 31 N. The background is $2 m$ LiDAR hillshade map, which is available at www.icgc.cat.

cerca de la cabecera, el valor de longitud tenderá a cero y el valor de la anomalía será bajo. Por lo tanto, aquellas estructuras ubicadas en las cabeceras de las cuencas, como inestabilidades de ladera y cambios litológicos, no se detectarán por el índice SL (Fig. 15). Asimismo, si el tramo analizado está alejado de la cabecera, la longitud respecto a ella será mayor y el índice destacará con valores más elevados las anomalías situadas aguas abajo. Además, en el caso particular de la cuenca del Romadriu, dado que la convexidad se acentúa más hacia la desembocadura, en ese tramo los valores de SL son aún mayores (Fig. 9).
La distancia del segmento $(d L)$ y la resolución del MDE son otros dos parámetros que influyen en el cálculo del índice SL. Debido a circunstancias técnicas y a las limitaciones temporales del trabajo, se decidió analizar las tres cuencas a partir de una distancia $d L=$ $50 \mathrm{~m}$ en el MDE de $5 \mathrm{~m}$ para optimizar el tiempo y poder obtener unos resultados preliminares. De cara a futuros proyectos, sería recomendable ampliar el análisis a las cuencas de Vall d'Àssua, Romadriu y Vall de Tor aplicando diversos valores de distancia de $d L$ con MDEs de mayor y menor resolución para estudiar la sensibilidad del método. 


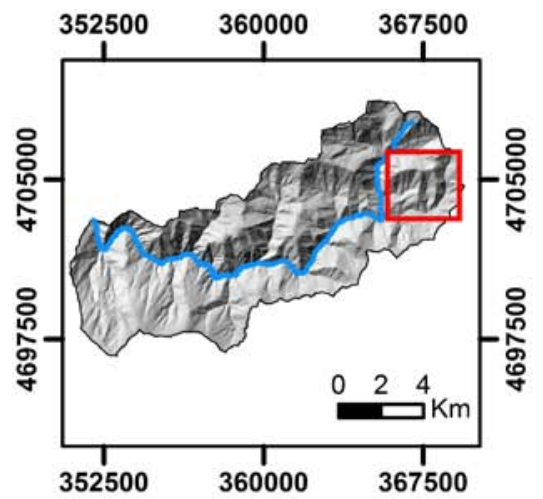

\section{Leyenda \\ Contactos \\ Contacto \\ indiferenciado}

Fallas

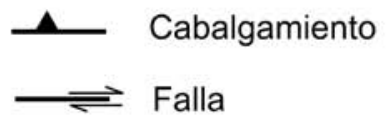

\section{Litología}

$\underline{\text { Cuaternario }}$

\section{Qac Depósitos aluviales (Holoceno). \\ Qco Depósitos coluviales (Holoceno)}

\section{Devónico}

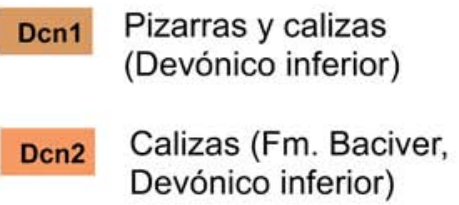

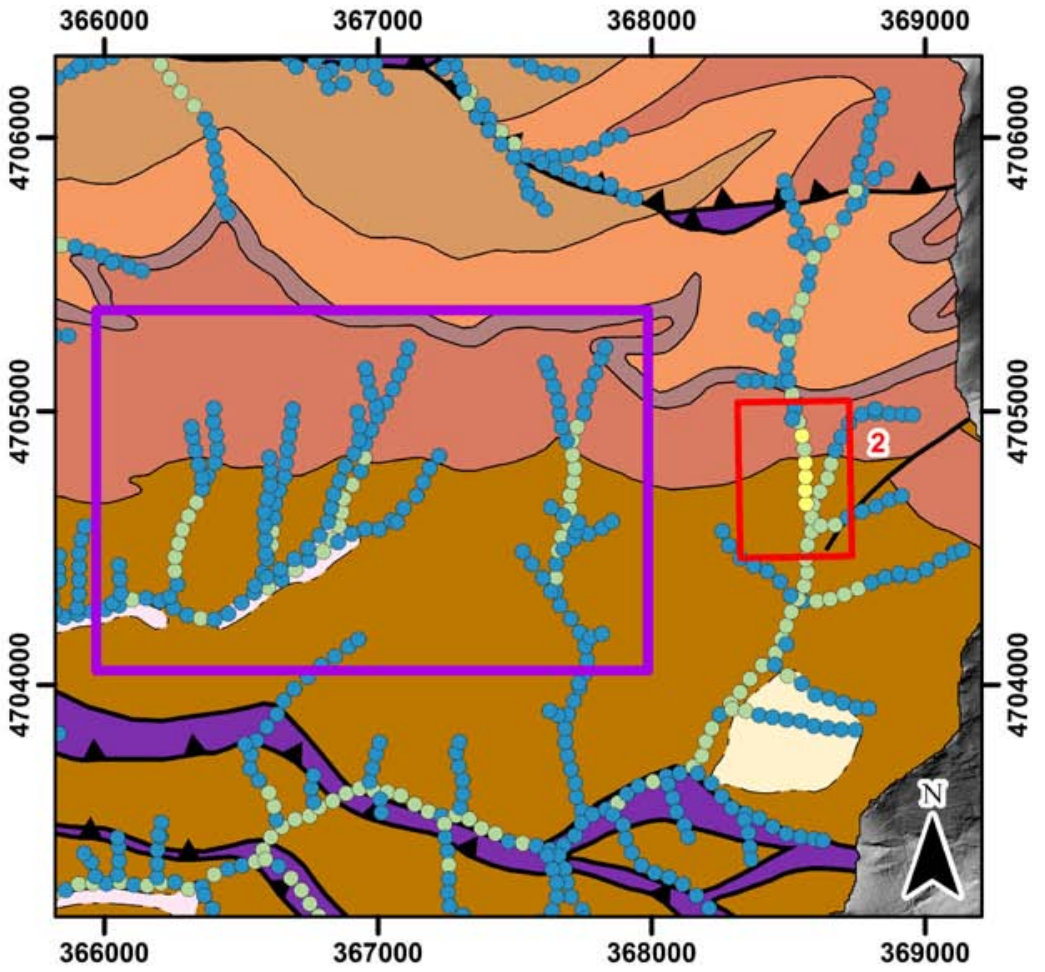

Der Calizas nodulosas (Fm. Manyanet, Devónico inferior - medio)

Valores del índice SL (m)

- $\quad<156$

- $\quad 156-451$

- $\quad 451-1000$

- $1000-2237$

- $\quad<2237$

\section{Anomalias}

(Fm. Fontjanina,

Devónico inferior).

\section{Silúrico}

SI Lutitas negras
(Silúrico)

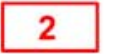

Zona asociada a un cambio litológico

Zona sin anomalia asociada a un cambio litológico

Figura 15. Ejemplo de la limitación del índice SL. El cambio litológico entre pizarras devónicas con las calizas devónicas no es destacado por los valores del índice SL cerca de la cabecera.

Figure 15. One example of the limitation of SL index. A lithological change between Devonian slates and limestone is not highlighted by the SL index near headwaters.

\section{Identificación e interpretación de anomalías}

En este trabajo se ha aplicado el método Troiani et al. (2017) basado en el análisis de Puntos Calientes y la Densidad de Kernel. Una vez localizadas las anomalías, en este proyecto, se ha realizado una visualización de la distribución del índice SL a lo largo del perfil longitudinal de un rio. Esta técnica ha ayudado a identificar las zonas que presentan los picos más altos del índice morfométrico. Además, el perfil se puede dividir en varios sectores caracterizados por un valor promedio de SL característico de cada tramo (Fig. 6). Otro criterio a utilizar es la caracterización de la anomalía a partir de un salto cualitativo importante entre un valor bajo y un valor alto de SL (Fig. 6).

No obstante, estas propuestas presentan varias 
limitaciones. En primer lugar, para dividir el perfil longitudinal en sectores, los valores de SL deben despuntar. Este rasgo sí sucede en las cuencas de La Vall $d$ 'Àssua y Romadriu, pero no en la Noguera de Tor de La Vall de Tor (Fig. 13A). En segundo lugar, caracterizar los tramos anómalos por un cambio brusco en valores de SL es muy costoso y no es recomendable si se requiere analizar en poco tiempo las anomalías importantes de una extensa región. En cualquier caso, en trabajos futuros se podría cuantificar este criterio de salto de valores del índice SL, para conseguir seleccionar anomalías de manera semiautomática.

Otras limitaciones presentes en este trabajo se encuentran en la interpretación de anomalías. Dada la baja actividad tectónica de la zona, la probabilidad de encontrar anomalías asociadas a fallas activas es baja, aunque para corroborar esta hipótesis se debería comparar las anomalías encontradas con un mapa detallado de fallas activas. También, utilizar el mapa geológico a escala 1:50.000 requiere una inspección de campo para comprobar aquellas anomalías producidas por cambios litológicos. Asimismo, ante la dificultad en la interpretación de las anomalías, se requiere un experto en inestabilidades de ladera y con conocimientos del contexto geodinámico de la región.

\section{Implicaciones para la mejora de inventarios de movi- mientos de ladera}

El análisis de anomalías del índice SL en las cuencas de La Vall d'Àssua, Romadriu y La Vall de Tor ha demostrado que en general hay una buena correlación entre anomalías y los movimientos de ladera. A grandes rasgos, entre las tres cuencas hay un total de 95 anomalías, de las cuales un $45 \%$ pueden estar relacionadas con movimientos en masa. El resto corresponde a factores indeterminados $(37 \%)$, a cambios litológicos $(14 \%)$ y a presas antrópicas $(2 \%)$.

Por lo tanto, estos resultados obtenidos muestran la utilidad del índice SL para identificar movimientos en masa. En primer lugar, permitiría elaborar inventarios preliminares en zonas no estudiadas a escala regional (1:200.000 - 1:50.000), similar al que se ha realizado en La Vall de Tor. En segundo lugar, este índice morfométrico podría ayudar y mejorar la identificación de movimientos en masa a escala local (1:50.000 - 1:10.000) como, por ejemplo, el caso de las inestabilidades de ladera de escala menor y mayor del complejo de Bordes de Llesui de La Vall d'Àssua. Y, finalmente, permitiría complementar inventarios ya existentes obtenidos a través de otras técnicas, como el de La Vall d'Àssua y el de Romadriu.
En cualquier caso, localizar estos knickpoints a lo largo del perfil del río permite identificar puntos de interés para estudiar la relación de estas anomalías con la posible presencia de movimientos en masa. Además, para comprobar si los knickpoints pueden identificar movimientos en masa que podrían estar activos, se debería realizar una inspección de campo para identificar rasgos que evidencien la actividad del movimiento de ladera, como escarpes bien conservados, fisuras o grietas en la cabecera o en el cuerpo del deslizamiento o deformaciones en construcciones antrópicas. También se podría tomar datos a partir de técnicas de auscultación como, por ejemplo, instalación de inclinómetros, GPS diferencial, DInSAR y estación total.

\section{Conclusiones}

Este trabajo ha permitido comprobar que el índice del gradiente del perfil longitudinal (Stream-Length Gradient Index, SL) es una técnica que permite analizar una región amplia y focalizar el estudio en aquellos puntos de la red de drenaje que están afectados por los movimientos en masa. En La Vall d'Àssua se ha visto una clara correlación espacial entre las anomalías y la mayoría de movimientos en masa cartografiados que alcanzan el fondo de valle, excepto en un caso en el torrente de Berasti. Además, se han identificado 13 nuevos movimientos en masa en zonas donde no habían sido descritos en La Vall de Tor, el Romadriu y La Vall d'Àssua.

En conclusión, este tipo de estudio morfométrico se ubicaría en la fase inicial del análisis de la peligrosidad: el inventario de los procesos. De este modo, se puede elaborar un primer inventario de una región no estudiada y, a partir de él, se puede hacer una evaluación preliminar de la susceptibilidad existente en una zona. Así, esta información puede servir de base para evaluar la peligrosidad. Sin embargo, el índice SL se ha de considerar como técnica complementaria a otras técnicas como fotointerpretación o la cartografía geomorfológica para ayudar a identificar estos fenómenos.

\section{Agradecimientos}

Este trabajo ha sido financiado por los Proyectos CHARMA (CGL2013-40828-R) y PROMONTEC (MINEICO-FEDER Ref.: CGL2017-84720-R). Quisiera agradecer a mis tutores, la Dra. Marta Guinau y el Dr. Jorge Pedro Galve, todo su apoyo, dedicación y paciencia. Gracias a los investigadores del Departament de 
Dinàmica de la Terra i l'Oceà (Facultat de Ciències de laTerra, Universitat de Barcelona) y del Departamento de Geodinámica (Facultad de Ciencias, Universidad de Granada) por sus aportaciones durante la realización del trabajo. Entre ellos una mención especial a María Ortuño por las horas de debate e ideas aportadas y a José Vicente Pérez-Peña por haber desarrollado y proporcionado la herramienta Chi-Map. Para acabar, mi agradecimiento también a Xavier Querol, del Institut Cartogràfic i Geològic de Catalunya (ICGC), por facilitarme los MDEs de alta resolución de las zonas de estudio.

\section{Referencias}

Bailey, C. The Longhill drainage ditch, when knickpoints move, 29/05/2017, http://wmblogs.wm.edu/.

Base de dades de moviments del terreny de Catalunya (LLISCAT). Caregue, 30/05/2017, http://siurana.icgc.cat/ Iliscat/.

Boissevain, H. 1934. Étude géologique et géomorphologique d'une partie de la haute vellée du Segre (Pyrénées Catalanes). Bulletin de la Societé d'Histoire Naturelle de Toulouse, LXVI, 32-170.

Capó, A. and García, C. 2014. Análisis e interpretación de los perfiles longitudinales en la red fluvial del Sur y Sureste de Mallorca. Cuadernos de Investigación Geográfica, 40, 147-167.

Castillo, M. and Lugo-Hubp, J. 2011. Estado actual del conocimiento, clasificación y propuesta de inclusión del término knickpoint en el léxico geológico-geomorfológico del español. Boletín de la Sociedad Geológica Mexicana, 23, 2, 353-364.

Clariana García, M.P. 2015. Estratigrafía, estructura y su relación con el metamorfismo de la zona axial pirenaica en la transversal del noroeste de Andorra y comarcas del Pallars Sobirá y el Alt Urgell (Lleida). Tesis Doctoral, Departamento de Geodinámica, Universidad de Oviedo, $221 \mathrm{pp}$.

Chen, Y.C., Sung, Q. and Cheng, K.Y. 2003. Along-strike variations of morphotectonic features in the Western Foothills of Taiwan: tectonic implications based on stream gradient and hypsometric analysis. Geomorphology, 56, 1-2, 109-137.

Confederación Hidrográfica del Ebro. IDE-Ebro (geodatos), 5/06/2017, http://ide-ebro.chebro.es/.

Corominas, J. and Alonso, E. 1984. Inestabilidad de Laderas en el Pirineo Catalán. Tipología y Causas. Jornadas de Trabajo sobre Inestabilidad de Laderas en el Pirineo. Barcelona, C1-C53.

El Hamdouni, R., Irigaray, C., Jiménez-Perálvarez, J.D. and Chacón, J. 2010. Correlations analysis between landslides and stream length-gradient (SL) index in the southern slopes of Sierra Nevada (Granada, Spain). In: Williams, A. L., Pinches, G.M., Chin, C.Y. McMorran, T.J., Massey, C.Y. (ed.), Geologically Active, London, 141-149. Environmental Systems Research Institute (ESRI-1). Análisis de punto caliente ( $\mathrm{Gi}^{*}$ de Getis-Ord), 31/05/2017, http://desktop.arcgis.com.

Environmental Systems Research Institute (ESRI-2). Densidad de Kernel, 31/05/2017, http://pro.arcgis.com.

Ermini, L. and Casagli, N. 2003. Prediction of the behaviour of landslide dams using a geomorphological dimensionless index. Earth Surface Processes and Landforms, 28, 31-47.

Furdada, G. 1988. Estudi geomorfològic de La Vall d'Àssua i marge dret de la Ribera de Sort. Tesina, Departament de Geologia Dinàmica, Geofísica i Paleontologia, Universitat de Barcelona, $182 \mathrm{pp}$.

Galve, J.P., Piacentini, D., Troiani, F. and Della Seta, M. 2014. Stream Length-Gradient Index Mapping as a Tool for Landslides Identification. In: Pardo-Igúzquiza E., Guardiola-Albert C., Heredia J., Moreno-Merino L., Durán J., Vargas-Guzmán J. (ed.), Mathematics of Planet Earth: Proceedings of the $15^{\text {th }}$ Annual Conference of the International Association for Mathematical Geosciences, Madrid, 343-346.

Getis, A. and Ord, J.K. 1992. The analysis of spatial association by use of distance statistics. Geographical Analysis, 24, 3, 189-206.

Goula, X., Olivera, C., Fleta, J., Grellet, B., Lindo, R., Rivera, L, Cisternas, A. and Carbon, D. 1999. Present and recent stress regime in the eastern part of the Pyrenees. Tectonophysics, 308, 487-502.

Hack, J.T. 1973. Stream-Profile Analysis and StreamGradient Index. Journal of Research of the United States Geological Survey, 1, 4, 421-429.

Hartevelt, J.J.A. 1970. Geology of the Upper Segre and Valira Valleys, Central Pyrenees, Andorra, Spain. Leidse Geologische Mededelingen, 45, 167-236.

Keller, E. A. and Pinter, N. 2002. Active Tectonics: Earthquakes, Uplift, and Landscape, Prentice Hall, Upper Saddle River, N. 228 pp.

Korup, O. 2004. Geomorphic hazard assessment of landslide dams in South Westland, New Zealand: fundamental problems and approaches. Geomorphology, 66, 167-188.

Lacan, P. and Ortuño, M. 2012. Active Tectonics of the Pyrenees: A review. Journal of Iberian Geology, 38, 1, 9-30.

Muñoz, J.A. 1992. Evolution of a continental collision belt: ECORS-Pyrenees crustal balanced crosssection. In: McClay, K.R. (ed.), Thrust Tectonics, Chapman y Hall, New York, 235-246.

Ortega, J.A. and Garzón, G. 2008. Cambios geomorfológicos en ríos en roca tras inundaciones de baja frecuencia (Río Girona, Alicante). Geogaceta, 44, 171-174.

Ortuño, M., Guinau, M., Calvet, J., Furdada, G., Bordonau, J., Ruíz, A. and Camafort, M. 2017. Potential of airborne LIDAR data analysis to detect subtle landforms of slope failure: the example of Portainé, Central Pyrenees. Geomorphology, 295, 364-382.

Ouimet, W. B., Whipple, K. X., Royden, L. H., Sun, Z. and Chen, Z. 2007. The influence of large landslides on river incision in a transient landscape: Eastern margin of the Tibetan Plateau (Sichuan, China). Geological Society of America Bulletin, 119, 11-12, 1462-1476.

Pánek, T., Smolková, V., Hradecky, J. and Kirchner, K. 2007. Landslide dams in the northern part of Czech flysch 
Carpathians: Geomorphic evidence and imprints. Studia Geomorphologica Carpatho-Balcanica, Landform evolution in mountain areas, $\mathrm{XLI}, 77-96$.

Silverman, B.W. 1986. Density Estimation for Statistics and Data Analysis. Chapman y Hall, Londres - Nueva York, 175 pp.

Troiani, F. and Della Seta, M. 2008. The use of the Stream Length-Gradient index in morphotectonic analysys of small catchments: A case study from Central Italy. Geomorphology, 102, 159-168.

Troiani, F., Galve, J.P., Piacentini, D., Seta, M.D. and Guerrero, J. 2014. Spatial analysis of stream length-gradient (SL) index for detecting hillslope processes: A case of the Gállego River headwaters (Central Pyrenees, Spain). Geomorphology, 214, 183-197.

Troiani, F., Piacentini, D., Seta, M.D. and Galve, J.P. 2017. Stream Length-gradient Hotspot and Cluster Analysis (SL-HCA) to fine-tune the detection and interpretation of knickzones on longitudinal profiles. Catena, 156, 30-41.
Van Den Eeckhaut, M. and Hervás, J. 2012. State of the art of national landslide databases in Europe and their potential for assessing landslide susceptibility, hazard and risk. Geomorphology, 139-140, 545-558.

Van Westen, C.J., Van Asch, T.W.J. and Soeters, R. 2006. Landslide hazard and risk zonation; why is it still so difficult? Bulletin of Engineering Geology and the Environment, 65, 2, 167-184.

Viveen, W., van Balen, R.T., Schoorl, J.M., Veldkamp, A., Temme, A.J.A.M. and Vidal-Romani, J.R. 2012. Tectonic geomorphology and neotectonic activity of the NWIberian Atlantic Margin with examples from the Miño river system. Tectonophysics, 544-545, 13-30.

Von Engeln, O.D. 1940. A particular case of knickpunkte. Annals of the Association of American Geographers, 30, 268-271.

Zandvliet, J. 1960. Geology of Upper Salat and Pallaresa Valleys, Central Pyrenees, France-Spain. Leidse Geologische Mededelingen, 25, 1-127.

Recibido: septiembre 2018

Revisado: febrero 2019

Aceptado: abril 2019

Publicado: septiembre 2019 
